\title{
Fermentation of Nocellara Etnea Table Olives by Functional Starter Cultures at Different Low Salt Concentrations
}

\author{
Alessandra Pino ${ }^{1}$, Maria De Angelis ${ }^{2}$, Aldo Todaro ${ }^{3}$, Koenraad Van Hoorde ${ }^{4}$, \\ Cinzia L. Randazzo ${ }^{1 *}$ and Cinzia Caggia ${ }^{1}$ \\ ${ }^{1}$ Department of Agricultural, Food and Environment, University of Catania, Catania, Italy, ${ }^{2}$ Department of Soil, Plant and Food \\ Sciences, University of Bari Aldo Moro, Bari, Italy, ${ }^{3}$ Department of Agricultural, Food and Forest Science, University of \\ Palermo, Palermo, Italy, ${ }^{4}$ Laboratory of Brewing and Biochemistry, Faculty of Bioscience Engineering, Ghent University, \\ Ghent, Belgium
}

OPEN ACCESS

Edited by:

Joaquin Bautista-Gallego, Instituto de la Grasa (IG), Spain

Reviewed by:

Rosanna Tofalo,

Università di Teramo, Italy

Konstantinos Papadimitriou, Agricultural University of Athens,

Greece

*Correspondence: Cinzia L. Randazzo cranda@unict.it

Specialty section:

This article was submitted to Food Microbiology,

a section of the journal

Frontiers in Microbiology

Received: 21 December 2017 Accepted: 14 May 2018

Published: 05 June 2018

Citation:

Pino A, De Angelis M, Todaro A, Van Hoorde K, Randazzo CL and Caggia C (2018) Fermentation of

Nocellara Etnea Table Olives by Functional Starter Cultures at Different Low Salt Concentrations. Front. Microbiol. 9:1125.

doi: 10.3389/fmicb.2018.01125
Nocellara Etnea is one of the main Sicilian cultivars traditionally used to produce both olive oil and naturally fermented table olives. In the present study, the effect of different salt concentrations on physico-chemical, microbiological, sensorial, and volatile organic compounds (VOCs) formation was evaluated in order to obtain functional Nocellara Etnea table olives. The experimental design consisted of 8 treatments as follow: fermentations at $4,5,6$, and $8 \%$ of salt with (E1-E4 samples) and without (C1-C4 samples) the addition of starters. All the trials were carried out at room temperature $\left(18 \pm 2^{\circ} \mathrm{C}\right)$ and monitored for an overall period of $120 \mathrm{~d}$. In addition, the persistence of the potential probiotic Lactobacillus paracasei N24 at the end of the process was investigated. Microbiological data revealed the dominance of lactic acid bacteria $(\llcorner A B)$, starting from the 7 th $d$ of fermentation, and the reduction of yeasts and enterobacteria in the final product inoculated with starters. VOCs profile highlighted a high amount of aldehydes at the beginning of fermentation, which significantly decreased through the process and a concomitant increase of alcohols, acids, esters, and phenols. In particular, esters showed an occurrence percentage higher in experimental samples rather than in control ones, contributing to more pleasant flavors. Moreover, acetic acid, ethanol, and phenols, which often generate off-flavors, were negatively correlated with mesophilic bacteria and LAB. It is interesting to note that salt content did not affect the performances of starter cultures and slightly influenced the metabolome of table olives. Sensory data demonstrated significant differences among samples registering the highest overall acceptability in the experimental sample at $5 \%$ of $\mathrm{NaCl}$. The persistence of the $L$. paracasei $\mathrm{N} 24$ strain in experimental samples, at the end of the process, revealed its promising perspectives as starter culture for the production of functional table olives with reduced salt content.

Keywords: $\mathrm{NaCl}$ content, probiotic strain, metabolomics, microbiota, REP-PCR analysis 


\section{INTRODUCTION}

The greater consumer's attention for healthy food is confirmed by the growing trend in fermented vegetables consumption, such as table olives (International Olive Council (IOC), 2016). Table olives are mainly produced in several Mediterranean countries, such as Spain, Italy, and Greece and in Sicily, two main cultivars (Nocellara del Belice and Nocellara Etnea) are growth. In particular, Nocellara Etnea cv is mainly cultivated in the Central and Eastern area of Sicily, among the provinces of Enna, Catania, Messina, Syracuse, and Ragusa. The drupes, elliptical in shape and slightly asymmetric, are characterized by a uniform and a large size and by late harvesting. The relationship between core and pulp is very high and this character makes this cultivar one of the best for the production of green table olives. The latter are mostly obtained by a spontaneous process in which the hydrolysis of oleuropein is relied on enzymatic activities of indigenous microorganisms, and on the plasmolytic effect of salt. This process is mainly dominated by lactic acid bacteria (LAB) and yeasts, which form a natural consortium (Randazzo et al., 2010). However, during the spontaneous fermentation spoilage microorganisms, such as Enterobacteriaceae and Propionibacteriaceae may occur. It is well established that Lactobacillus plantarum and Lactobacillus pentosus are the main detected species, due to their versatile adaptation to the brine environment (Ruiz-Barba et al., 1994; GAlegria et al., 2004; Bautista-Gallego et al., 2010; Randazzo et al., 2011, 2012; Hurtado et al., 2012; Cocolin et al., 2013; Tofalo et al., 2014), and different strains are widely used as starter cultures in several table olive fermentations (Arroyo-López et al., 2012; Hurtado et al., 2012; Randazzo et al., 2014).

During olive fermentation, coarse salt is added in order to reduce the water activity, preventing the growth of spoilage microorganisms, and to improve taste and textures of the final product (Bautista-Gallego et al., 2013a). The EU Member States try to implement national nutritional policies with the aim to decrease salt intake according to the European Commission suggestion (European Council, 2010). The strategy to set up table olives with a reduced daily $\mathrm{Na}$ intake, which has been established at $5 \mathrm{~g}$ salt, by WHO/FAO [World Health Organisation (WHO)/Food 2003] is one of the main goals of food industry. Different chloride salts, such as $\mathrm{KCl}, \mathrm{CaCl}_{2}$, and $\mathrm{ZnCl}_{2}$, have been evaluated as replacers for $\mathrm{NaCl}$ (Bautista-Gallego et al., 2013b), especially in Spanish style green olives (Bautista-Gallego et al., 2010, 2011). The reduction in $\mathrm{Na}$ and the increase in other salts may lead to a more equilibrated mineral composition in table olives, ameliorating the consumers' diet, and enhancing the perception of the nutritional value of the olives. Nevertheless, the effects of $\mathrm{NaCl}$ replacement with other salts could affect the microbiota evolving in the fermentation process of table olives (Bautista-Gallego et al., 2015; Mateus et al., 2016), as well as impact the sensorial quality of the final product (Zinno et al., 2017). Potential $\mathrm{NaCl}$ reduction depends on characteristics linked with the cultivar, its composition, other ingredients, processing, and technological parameters (BautistaGallego et al., 2013a), which should be well addressed before their implementation at the industrial scale. Furthermore, the final product must be safe from the microbiological point of view. It is already established that a reduction in $\mathrm{NaCl}$ might be responsible for an increase of pathogens such as Clostridium botulinum (Simpson et al., 1995).

Nowadays, based on the increasing consumers' demand, the production of healthier table olives is of great industry importance, taking into account the potential market of table olives as a functional food. In fact, functional table olives can provide a concrete opportunity to convey the benefits that are already appreciated by consumers in dairy sectors. It has already been demonstrated that table olives represent a good vehicle to transport probiotics to humans for both their microarchitecture and the presence of nutrients (Lavermicocca et al., 2005; Valerio et al., 2006; Randazzo et al., 2017).

The aim of the present study was (i) to set up a fermentation, at laboratory scale, of Nocellara Etnea table olives with reduced level of $\mathrm{NaCl}$; (ii) to evaluate the effect of the $\mathrm{NaCl}$ reduction on the physico-chemical, microbiological, and sensorial parameters compared to fermentation carried out without starter cultures.

\section{MATERIALS AND METHODS}

\section{Bacterial Strains and Olive Processing}

In the present study two lyophilized LAB strains, L. plantarum UT2.1 and the potential probiotic Lactobacillus paracasei N24, belonging to the Di3A microbial collection, previously screened for their technological and functional features, and already applied as starter cultures at industrial scale (Randazzo et al., 2017) were used. Each strain was directly inoculated into fresh brine (1:1 ratio) to reach a final cell density of 7 log colony forming units per $\mathrm{ml}(\mathrm{cfu} / \mathrm{ml})$. Olives of Nocellara Etnea cultivar, kindly provided by a local company (Consoli srl, Adrano, Sicily) were processed, at laboratory scale, following the Sicilian style method. After harvesting, about $3 \mathrm{~kg}$ of olives were subjected to quality control, to remove damaged fruits, washed with tap water, directly placed in sterile glass vessels, and covered with $\sim 31$ of sterile brine. The experimental design consisted of 8 treatments as follows: fermentations at 4, 5, 6, and $8 \%$ of salt with the addition of starters (E1-E4 samples); fermentations at $4,5,6$, and $8 \%$ of salt without the addition of starters (C1C4 samples). All fermentation trials were carried out at room temperature $\left(18 \pm 2^{\circ} \mathrm{C}\right)$, and monitored for an overall period of $120 \mathrm{~d}$. The brine salt concentration was maintained at each initial level by adding marine salt. Fresh brine was periodically supplied to maintain olives totally dipped in order to inhibit growth of molds on the brine surfaces. The experimental trials were carried out in triplicate. The progression of the fermentation was followed by monitoring $\mathrm{pH}$, titratable acidity and the shift in microbial populations in brine throughout the process.

\section{Physico-Chemical and Total Polyphenol Determination of Brine Samples}

Fifty $\mathrm{ml}$ of each brine sample were taken at 60 and 120 days of fermentation. The $\mathrm{pH}$ values of brines were monitored by a $\mathrm{pH}$ meter (H19017, Microprocessor, Hanna Instruments). Total free acidity was measured by titration and expressed as the percentage of lactic acid ( $\mathrm{g} / 100 \mathrm{ml}$ brine). Total polyphenol content was 
colorimetrically determined in brine samples at 60 and $120 \mathrm{~d}$, using Folin-Ciocalteu reagents, according to Singleton (1974). Polyphenols were measured in triplicate and expressed as $\mathrm{mg} / \mathrm{l}$ of gallic acid.

\section{Microbiological Analyses of Brine Samples}

For the microbiological characterization, brine samples were analyzed at $1,7,15,30,60,90$, and 120 days of fermentation. At each sampling time, brines were serially diluted, using sterile quarter-strength Ringer's solution (QRS), and plated in triplicate on the following agar media (all provided from Oxoid Italy), and conditions: Plate Count Agar, incubated at $32 \pm 2^{\circ} \mathrm{C}$ for $48 \mathrm{~h}$, for total mesophilic bacteria; de Man-Rogosa-Sharp agar, supplemented with cycloheximide ( $5 \mathrm{ml} / \mathrm{l}$ ), anaerobically incubated at $32^{\circ} \mathrm{C}$ for $24-48 \mathrm{~h}$, for LAB count; Sabouraud Dextrose Agar, supplemented with chloramphenicol $(0.05 \mathrm{~g} / \mathrm{l})$, incubated at $25^{\circ} \mathrm{C}$ for 4 days, for yeast count; Violet Red Bile Glucose Agar, aerobically incubated at $37^{\circ} \mathrm{C}$ for $24 \mathrm{~h}$, for Enterobacteriaceae count; Mannitol Salt Agar, incubated at $32^{\circ} \mathrm{C}$ for $48 \mathrm{~h}$, for staphylococci enumeration; Mac Conkey incubated at $32^{\circ} \mathrm{C}$ for $24-48 \mathrm{~h}$ for Escherichia coli determination. Results were expressed as $\log _{10}$ $\mathrm{cfu} / \mathrm{ml}$.

\section{Volatile Organic Compound (VOC) Analysis by Gas Chromatography-Mass-Spectrometry (GC-MS)}

VOCs, detected in brine samples at 1, 60, and 120 $\mathrm{d}$ of fermentation, were sampled using a solid-phase microextraction (SPME). The SUPELCO SPME (Bellefonte, PA) fiber holder and fiber used were coated with divinylbenzene/polydimethylsiloxane (DV/PDMS), $65 \mathrm{~mm}$. Before the first extraction, the fiber was conditioned in the GC injector port at $300^{\circ} \mathrm{C}$ for $1 \mathrm{~h}$, according to the manufacturer's recommendation. Ten $\mathrm{ml}$ of brine sample were added to a $35 \mathrm{ml}$ vial. Extraction temperature of head-space and time were $40^{\circ} \mathrm{C}$ and $20 \mathrm{~min}$, respectively. One $\mathrm{g}$ of $\mathrm{NaCl}$ was added to increase extraction rate of VOCs. The samples were gently vortexed during extraction using a magnetic stirrer. Fiber exposition was prolonged for $20 \mathrm{~min}$ at $40^{\circ} \mathrm{C}$. Thermal desorption was performed in the injector at $230^{\circ} \mathrm{C}$ for $1 \mathrm{~min}$ (Sabatini et al., 2008; Malheiro et al., 2011). The identification of the extracted VOCs was carried out using a GC instrument (HP GC6890, Hewlett Packard, Palo Alto, CA), coupled to a MS detector (HP MS5973) (Panagou and Tassou, 2006). The gas chromatograph was equipped with a $30 \mathrm{~m} 0.25 \mathrm{~mm}$ i.d. $0.25 \mathrm{~mm}$ film thickness fused-silica capillary column (DB-WAX J\&W Scientific) and the injector temperature was $230^{\circ} \mathrm{C}$. The conditions applied were as those previously reported (Randazzo et al., 2017). The quantification of VOCs was determined with the internal standard method spiking propionic acid, ethanol, ethyl acetate, benzaldehyde and guaiacol to all analyzed samples. All analyses were performed in duplicate and the results were expressed in $\mu \mathrm{g} / \mathrm{l}$ of brine.

\section{Isolation and Genetic Identification of Lactic Acid Bacteria}

From each MRS agar plate, at the highest dilution, of both $\mathrm{E}$ (E1-E4) and $\mathrm{C}(\mathrm{C} 1-\mathrm{C} 4)$ brine samples at 1, 60, and 120 $\mathrm{d}$ of fermentation, the $20 \%$ of total number of colonies was randomly selected, purified, checked for catalase activity and Gram reaction, and microscopically examined before storing in liquid culture using $20 \%$ glycerol at $-80^{\circ} \mathrm{C}$. The random colony selection from the highest dilution plates allowed us to collect $400 \mathrm{LAB}$ isolates. Total genomic DNA of isolates was extracted from overnight cultures according to the method described by Pino et al. (2018). DNA concentration and DNA quality were assessed by measuring optical density using Fluorometer Qubit (Invitrogen, Carlsbad, 278 CA, USA).

All LAB isolates were subjected to RecA and Tuf gene speciesspecific PCR following the protocol previously described by (Torriani et al., 2001; Ventura et al., 2003).

\section{REP-PCR Analysis}

In order to evaluate the viability of the potential probiotic L. paracasei N24 throughout the fermentation, lactobacilli isolated from E samples at 60 and $120 \mathrm{~d}$ (60 isolates), ascribed to $L$. paracasei species through the aforementioned speciesspecific multiplex PCR, were subjected to REP-PCR analysis, using the (GTG) ${ }_{5}$-primer, as described by Gevers et al. (2001). PCR amplicons were separated on a $1.5 \%$ agarose gel $(\mathrm{w} / \mathrm{v})$ in $1 \mathrm{X}$ TAE buffer (40 mM Tris, $20 \mathrm{mM}$ acetic acid and $1 \mathrm{mM}$ EDTA) under highly standardized conditions ( $55 \mathrm{~V}, 400 \mathrm{~mA}, 16 \mathrm{~h}$ at $\left.4^{\circ} \mathrm{C}\right)$. At regular intervals a reference marker $(6 \mu \mathrm{l}$ each composed of $1.10 \mu \mathrm{l}$ Molecular Ruler 500 bp (Bio-Rad), $1.40 \mu \mathrm{l}$ Molecular Ruler 100 bp (Bio-Rad), $2 \mu$ l TE buffer (1 mM EDTA, $10 \mathrm{mM}$ Tris-HCl, $\mathrm{pH}$ 8.0) and $1.50 \mu \mathrm{l}$ loading dye), was loaded for normalization. Profiles were visualized under ultraviolet light, after staining with ethidium bromide. Digitized images of gels were normalized and analyzed by the BioNumerics 7.6.2 software (Applied Maths, Belgium). Similarity matrices of densitometric curves of the gel tracks were calculated with Pearson's productmoment correlation coefficient. Subsequent cluster analyses of similarity matrices were performed by unweighted pair group method with arithmetic averages (UPGMA).

\section{Table Olives Sensory Evaluation}

The sensory assessment of table olives was performed by a trained sensory panel consisting of 10 panelists ( 6 females and 4 males, aged from 22 to 40 years), according to the method reported by the International Olive Council (International Olive Council (IOC), 2011). Olives were tasted for negative sensations (abnormal fermentation such as musty, rancid, cooking effect, soapy, metallic, earthy, and winey-vinegary), based on the classification reported by International Olive Council (IOC) (2011, 2016), while a descriptive analysis was carried out for descriptors corresponding to gustatory sensations (acidity, saltiness, and bitterness) and kinaesthetic sensations (hardness, fibrousness, and crunchiness). In addition, an overall acceptability descriptor was considered as an indication of the overall quality. Sensory data were acquired by a direct computerized registration system (FIZZ Biosystemes. Couternon, France). 


\section{Statistical Analysis}

Statistical analysis of chemical data was performed using a one-way analysis of variance with repeated measures of the GLM procedure by SAS (2001), considering the different treatments as variable. Means were separated by a Least Significant Difference (LSD) test when a significant treatment $(P<0.05)$ was observed. Microbiological data and VOCs were analyzed by ANOVA (One-way Analysis of Variance) using Tukey's post-hoc test, in order to assess the overall differences among samples. Statistical analysis was performed using XLSTAT PRO 5.7 (Addinsoft, New York, USA) and the reference level of significance was 0.05 in all the assays. Sensory data were submitted to one-way ANOVA using the software package Statgraphics ${ }^{\circledR}$ Centurion XVI (Statpoint Technologies, INC.) using samples as treatments. The significance was tested by means of the F-test. To differentiate the samples, the mean values were submitted to the multiple comparison test using the LSD procedure. In order to correlate the

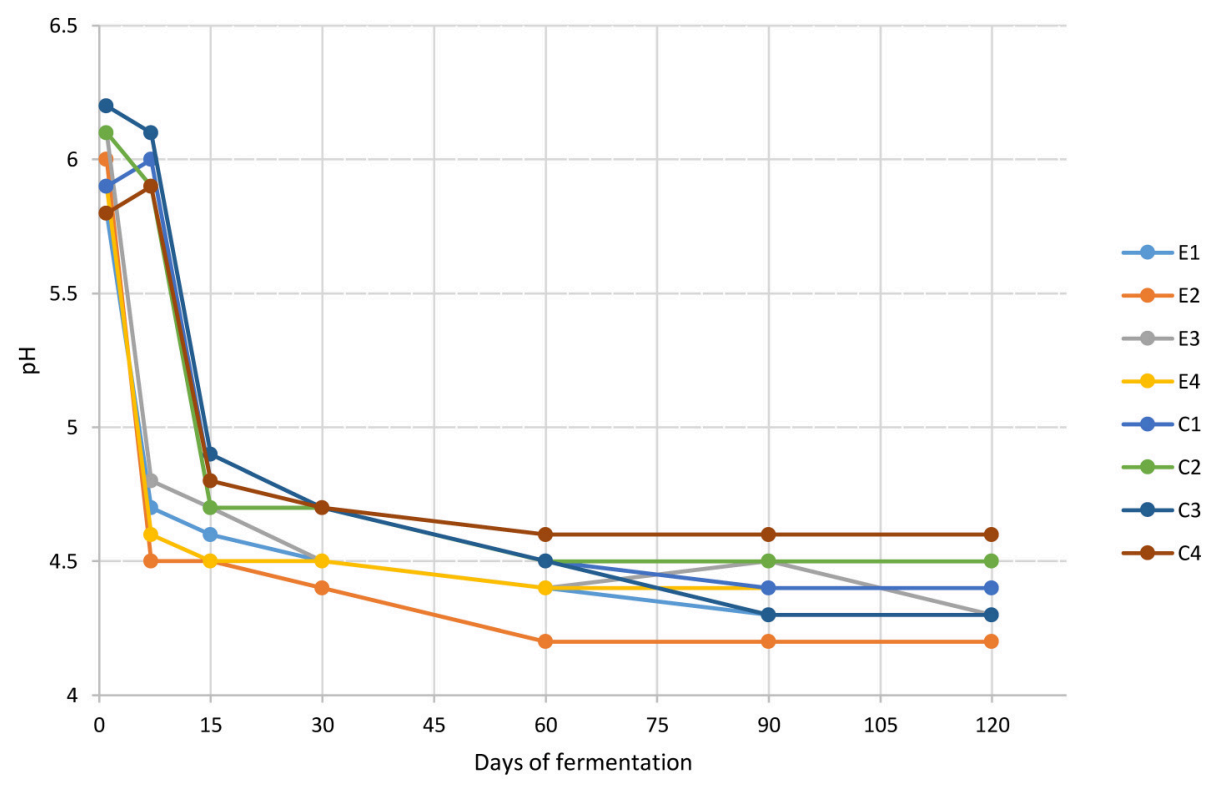

FIGURE 1 | Changes in pH throughout the fermentation of Nocellara Etnea experimental and control samples

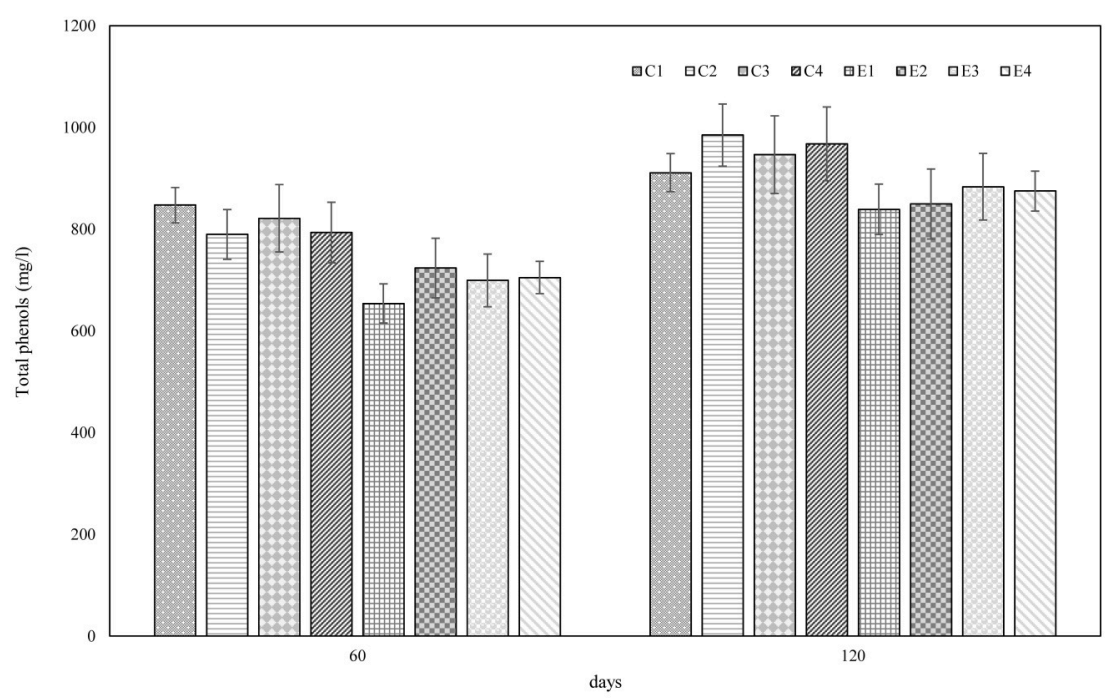

FIGURE 2 | Total phenolic content (mg/l) of control (C) experimental (E) brine samples at 60 and 120 days of fermentation. 
experimental and control brine samples to volatile compounds, data obtained at 1,60 , and $120 \mathrm{~d}$ of fermentation were subjected to principal component analysis (PCA) using MATLAB, achieving high data compression efficiency of the original data.

Similarities between the microbiota and metabolome profiles of experimental and control brine samples were carried out by PermutMatrix software. Data correlations between microbiota (mesophilic bacteria, LAB, staphylococci, yeasts, enterobacteria) and VOCs were computed using Statistica v. 7.0 and elaborated through PermutMatrix software.

\section{RESULTS}

\section{Physico-Chemical Data}

The $\mathrm{pH}$ values, showed in Figure 1, dropped faster in experimental samples rather than in control ones reaching a value between 4.5 and 4.8 at 7 days of fermentation. The lowest $\mathrm{pH}$ value (4.2) was revealed by E2 sample at 60 days. At the end of the process, $\mathrm{pH}$ values ranged from 4.6 to 4.2 . The titratable acidity, expressed as the percentage of lactic acid ( $\mathrm{g} / 100 \mathrm{ml}$ brine), was determined at 60 and $120 \mathrm{~d}$ of fermentation. Overall, no differences were detected among experimental and control samples and results showed an increase during the fermentation process, reaching an average value of $0.37 \mathrm{~g} / 100 \mathrm{ml}$ brine in the final products (data not shown). Results of total phenols (TP) content are presented in Figure 2. TP detected in brines showed quite differences among $\mathrm{E}$ and $\mathrm{C}$ samples while within each group similarities on TP content was achieved. In addition, statistically significant differences were noticed between samples at 60 and $120 \mathrm{~d}$ of fermentation with a range of 911.2-985.2 mg/l for C samples and of 839.2-967.8 mg/l for E samples.

\section{Microbial Counts of Brine Samples Through the Fermentation}

Microbial counts, expressed as $\log _{10} \mathrm{cfu} / \mathrm{ml}$, of both experimental (E) and control (C) brine samples, at different salt concentrations $(4,5,6$, and $8 \%)$, are reported in Table 1. Viable mesophilic bacteria exhibited different behavior among samples. In detail, a steady trend in samples inoculated with starter cultures (E1E4), was recorded, with the exception of E4 sample (at $8 \%$ of $\mathrm{NaCl}$ ), in which, from a lower initial value (6.65 log unit), a slight decrease throughout the fermentation was observed. Control samples (C1-C4) exhibited a mean initial value of 4.6 $\pm 0.07 \log$ unit, which significantly increased from the 15 th day of fermentation, reaching a final mean value of $5.43 \pm$ 0.09 log unit (Table 1). Similar behavior was observed for LAB population, which reached the highest concentration value in all inoculated samples (E1-E4). The highest value (8.58 log unit) was detected after 60 days of fermentation in sample E2, obtained adding $5 \%$ of salt. Among the control samples (C1C4), a similar initial LAB count was found (average value of $4.4 \pm 0.20 \log \mathrm{cfu} / \mathrm{ml}$ ) with the exception of $\mathrm{C} 4$ sample, which presented the lowest LAB cell density (Table 1). At the end of the fermentation (120 days) no statistically significant differences $(P>0.05)$ were achieved among samples, that exhibited an average value of $5.88 \pm 0.15 \log \mathrm{cfu} / \mathrm{ml}$. Yeasts were present at an initial average level of $3.93 \pm 0.08 \mathrm{log} \mathrm{cfu} / \mathrm{ml}$ and $4.18 \pm$ $0.17 \log \mathrm{cfu} / \mathrm{ml}$, in $\mathrm{E}$ and $\mathrm{C}$ samples, respectively. These densities slightly increased through the fermentation process, achieving, at 120 days, an average value of $5.51 \pm 0.13 \mathrm{log}$ unit, with the exception of samples E1, E2, and E3, which exhibited the lowest yeast count (Table 1). At the beginning of fermentation, the staphylococci level was quite similar among inoculate and un-inoculated samples, with a slight increase at 30 and 60 days, and followed by a decrease to a final average value of $3.53 \pm$ $0.19 \mathrm{cfu} / \mathrm{ml}$. Enterobacteria counts significantly decreased from the 30th day of fermentation, reaching a final value below 2 log. In addition, E. coli was never detected in any brine samples analyzed.

\section{Volatile Organic Compound (VOC) Detection by Gas Chromatography-Mass-Spectrometry (GC-MS)}

Volatile organic compounds (VOCs) of $\mathrm{E}$ and C brine samples at 1, 60, and $120 \mathrm{~d}$ of fermentation, are reported in Table 2. The assessment allowed the identification of 47 compounds as acids, alcohols, esters, aldehydes, and phenols. Overall, total VOCs exhibited a growing trend during the fermentation reaching an average value of $2349.70 \mu \mathrm{g} / \mathrm{l}$ after 120 days. In particular, the highest values were registered in all control samples, with a mean value of $3215.23 \mu \mathrm{g} / \mathrm{l}$. In detail, in all samples, at beginning of fermentation, aldehydes represented the main VOCs, and after 60 days, they significantly decreased, whereas alcohols, acids, esters and phenols increased. At the end of fermentation (120 d), differences were observed among brine samples. Zooming on each chemical class, it is possible to assert that overall, the detected amounts of each compounds were sample-dependent. Among acids, the acetic acid was the most abundant compound, with the highest values in control samples, whereas hexanoic and propionic acids were more abundant in experimental samples (Table 2). Among alcohols, ethanol dominated the fermentation process, especially in control samples, followed by isoamyland phenyl-ethyl alcohol. Among esters, the highest amount was achieved by ethyl-acetate, followed by ethyl lactate. A different trend was revealed for butanoic-acid-2-methylester, which showed the highest value in the E2 sample, followed by control samples. The most abundant aldehyde and phenols were nonanal and cresol, respectively. Evaluating the VOCs occurrence percentage on $\mathrm{E}$ and $\mathrm{C}$ samples at 120 days of fermentation (Figure S1), it is interesting to note that esters and acids were mainly present in all $\mathrm{E}$ samples with the highest occurrence percentage in E2 (31.7 and 11.3\%, respectively). Alcohols, phenols, and aldehydes were also detected at high occurrence percentage in E2 sample (Figure S1). Figures 3A,B showed the PCA plot of distribution of C (C1-C4) and E (E1E4) samples, at different days of fermentation, in the PC1-PC2 plane. Based on the loadings (data not shown), component 1 , that represent the $80.88 \%$ of the variability, can be viewed as an esters factor, while the second principal component (variance 11.64\%), 
TABLE 1 | Microbial counts expressed as $\log _{10}$ CFU/ml of 3 replicates \pm standard deviation of the main microbial groups detected in Nocellara Etnea table olives at different salt concentrations (4, 5, 6, 8\%) trough experimental (E) and spontaneous (C) fermentation (1 to 120 days).

\begin{tabular}{|c|c|c|c|c|c|c|c|}
\hline \multicolumn{8}{|c|}{ DAYS OF FERMENTATION } \\
\hline & 1 & 7 & 15 & 30 & 60 & 90 & 120 \\
\hline \multicolumn{8}{|c|}{ MESOPHILIC BACTERIA } \\
\hline & $7.36 \pm 0.05^{d}$ & $7.75 \pm 0.06^{9}$ & $7.53 \pm 0.20^{\mathrm{e}}$ & $7.74 \pm 0.05^{e}$ & $7.44 \pm 0.18^{d}$ & $7.15 \pm 0.12^{d}$ & $7.35 \pm 0.08^{f}$ \\
\hline \multicolumn{8}{|l|}{ E1 } \\
\hline E2 & $7.31 \pm 0.06^{d}$ & $7.25 \pm 0.30^{f}$ & $7.51 \pm 0.06^{\mathrm{e}}$ & $7.01 \pm 0.12^{d}$ & $7.40 \pm 0.10^{d}$ & $7.11 \pm 0.14^{d}$ & $7.01 \pm 0.08^{\mathrm{ef}}$ \\
\hline E3 & $7.23 \pm 0.09^{d}$ & $6.95 \pm 0.43^{\mathrm{e}}$ & $7.49 \pm 0.06^{e}$ & $6.99 \pm 0.02^{d}$ & $6.65 \pm 0.09^{c}$ & $6.93 \pm 0.10^{d}$ & $7.05 \pm 0.14^{\mathrm{e}}$ \\
\hline E4 & $6.56 \pm 0.11^{\mathrm{C}}$ & $6.75 \pm 0.18^{d}$ & $6.53 \pm 0.24^{d}$ & $5.94 \pm 0.41^{\mathrm{C}}$ & $5.12 \pm 0.37^{a}$ & $5.82 \pm 0.24^{b}$ & $5.73 \pm 0.13^{c}$ \\
\hline C1 & $4.21 \pm 0.06^{a}$ & $4.40 \pm 0.21^{a}$ & $4.83 \pm 0.33^{a}$ & $5.20 \pm 0.09^{a}$ & $5.11 \pm 0.11^{\mathrm{a}}$ & $5.36 \pm 0.08^{a}$ & $5.07 \pm 0.05^{a}$ \\
\hline $\mathrm{C} 2$ & $4.38 \pm 0.10^{a}$ & $4.53 \pm 0.15^{a b}$ & $5.02 \pm 0.21^{b}$ & $5.92 \pm 0.09^{c}$ & $5.36 \pm 0.07^{a b}$ & $5.60 \pm 0.06^{b c}$ & $5.39 \pm 0.06^{b}$ \\
\hline C3 & $4.89 \pm 0.09^{b}$ & $4.81 \pm 0.05^{\mathrm{ac}}$ & $5.51 \pm 0.18^{c}$ & $5.97 \pm 0.11^{\mathrm{C}}$ & $5.45 \pm 0.09^{a b}$ & $5.40 \pm 0.12^{\mathrm{ac}}$ & $5.53 \pm 0.13^{b c}$ \\
\hline $\mathrm{C} 4$ & $4.82 \pm 0.03^{b}$ & $4.68 \pm 0.18^{a}$ & $5.21 \pm 0.21^{b}$ & $5.68 \pm 0.28^{b}$ & $5.63 \pm 0.12^{b}$ & $5.58 \pm 0.23^{\mathrm{ac}}$ & $5.74 \pm 0.12^{\mathrm{cd}}$ \\
\hline \multicolumn{8}{|c|}{ LACTIC ACID BACTERIA } \\
\hline & $7.09 \pm 0.10^{d}$ & $7.52 \pm 0.12^{\mathrm{g}}$ & $8.08 \pm 0.259$ & $8.72 \pm 0.15^{f}$ & $8.13 \pm 0.12^{\mathrm{e}}$ & $7.85 \pm 0.13^{d}$ & $7.83 \pm 0.07^{\mathrm{de}}$ \\
\hline \multicolumn{8}{|l|}{ E1 } \\
\hline E2 & $7.12 \pm 0.10^{\mathrm{de}}$ & $7.59 \pm 0.139$ & $7.38 \pm 0.30^{d}$ & $8.27 \pm 0.07^{e}$ & $8.49 \pm 0.05^{d}$ & $8.58 \pm 0.08^{e}$ & $8.23 \pm 0.11^{d}$ \\
\hline E3 & $7.02 \pm 0.21^{\mathrm{de}}$ & $7.14 \pm 0.05^{f}$ & $7.15 \pm 0.35^{\mathrm{de}}$ & $7.03 \pm 0.10^{b}$ & $7.64 \pm 0.07^{c}$ & $7.86 \pm 0.11^{d}$ & $7.79 \pm 0.15^{\mathrm{de}}$ \\
\hline E4 & $7.03 \pm 0.11^{\mathrm{e}}$ & $7.37 \pm 0.27^{e}$ & $7.34 \pm 0.09 \mathrm{df}$ & $7.02 \pm 0.21^{a b c}$ & $7.30 \pm 0.40^{b}$ & $7.05 \pm 0.53^{c}$ & $7.04 \pm 0.30^{c}$ \\
\hline $\mathrm{C} 1$ & $4.51 \pm 0.20^{a b c}$ & $4.69 \pm 0.13^{b}$ & $5.81 \pm 0.05^{\mathrm{C}}$ & $7.36 \pm 0.11^{\mathrm{cd}}$ & $7.83 \pm 0.05^{c}$ & $6.88 \pm 0.13^{b}$ & $6.00 \pm 0.08^{a}$ \\
\hline $\mathrm{C} 2$ & $4.48 \pm 0.23^{C}$ & $4.79 \pm 0.18^{b c}$ & $5.96 \pm 0.07^{c}$ & $7.21 \pm 0.07^{\mathrm{ad}}$ & $7.32 \pm 0.06^{b}$ & $6.04 \pm 0.09^{a}$ & $5.96 \pm 0.11^{a}$ \\
\hline C3 & $4.21 \pm 0.16^{b}$ & $4.57 \pm 0.09^{b d}$ & $5.53 \pm 0.13^{b}$ & $7.10 \pm 0.13^{\mathrm{ad}}$ & $6.74 \pm 0.12^{a}$ & $6.78 \pm 0.10^{\mathrm{b}}$ & $5.67 \pm 0.06^{a}$ \\
\hline C4 & $3.93 \pm 0.21^{a}$ & $3.85 \pm 0.09^{a}$ & $5.12 \pm 0.21^{a}$ & $6.95 \pm 0.35^{\mathrm{a}}$ & $6.58 \pm 0.14^{a}$ & $5.96 \pm 0.42^{a}$ & $5.94 \pm 0.36^{a}$ \\
\hline \multicolumn{8}{|c|}{ YEASTS } \\
\hline & $4.00 \pm 0.01^{d}$ & $4.63 \pm 0.13^{\mathrm{C}}$ & $5.27 \pm 0.28^{a}$ & $5.13 \pm 0.06^{a}$ & $5.47 \pm 0.18^{a}$ & $5.20 \pm 0.12^{a b}$ & $4.61 \pm 0.05^{b}$ \\
\hline \multicolumn{8}{|l|}{ E1 } \\
\hline E2 & $3.97 \pm 0.10^{\mathrm{C}}$ & $4.18 \pm 0.06^{b}$ & $5.35 \pm 0.31^{a}$ & $5.61 \pm 0.09^{a}$ & $5.56 \pm 0.11^{a}$ & $5.60 \pm 0.15^{b}$ & $4.11 \pm 0.06^{a}$ \\
\hline E3 & $3.84 \pm 0.01^{a}$ & $5.05 \pm 0.14^{d}$ & $5.62 \pm 0.31^{a b}$ & $5.28 \pm 0.07^{a}$ & $5.51 \pm 0.10^{a}$ & $5.14 \pm 0.11^{a}$ & $4.30 \pm 0.05^{a b}$ \\
\hline E4 & $3.93 \pm 0.21^{b}$ & $3.46 \pm 0.11^{a}$ & $5.81 \pm 0.18^{b}$ & $5.23 \pm 0.21^{a}$ & $5.68 \pm 0.30^{a}$ & $5.12 \pm 0.24^{a}$ & $5.50 \pm 0.23^{C}$ \\
\hline C1 & $4.21 \pm 0.12^{\mathrm{g}}$ & $5.34 \pm 0.30^{d}$ & $6.73 \pm 0.18^{\mathrm{C}}$ & $6.25 \pm 0.12^{b}$ & $6.28 \pm 0.10^{b}$ & $5.32 \pm 0.09^{a b}$ & $5.58 \pm 0.09^{c}$ \\
\hline $\mathrm{C} 2$ & $4.28 \pm 0.23^{\mathrm{bcd}}$ & $6.12 \pm 0.32^{e}$ & $6.61 \pm 0.21^{c}$ & $6.85 \pm 0.11^{\mathrm{c}}$ & $6.48 \pm 0.11^{\mathrm{bc}}$ & $5.28 \pm 0.14^{a b}$ & $5.72 \pm 0.08^{\mathrm{C}}$ \\
\hline C3 & $4.09 \pm 0.09^{e}$ & $5.03 \pm 0.11^{d}$ & $5.61 \pm 0.11^{a b}$ & $6.16 \pm 0.08^{b}$ & $5.47 \pm 0.05^{a b}$ & $5.00 \pm 0.09^{a}$ & $5.36 \pm 0.14^{c}$ \\
\hline C4 & $4.14 \pm 0.23^{f}$ & $4.96 \pm 0.16^{\mathrm{cd}}$ & $5.78 \pm 0.23^{a b}$ & $5.63 \pm 0.31^{a}$ & $5.91 \pm 0.26^{a b}$ & $5.32 \pm 0.27^{a b}$ & $5.40 \pm 0.11^{c}$ \\
\hline \multicolumn{8}{|c|}{ STAPHYLOCOCCI } \\
\hline & $4.76 \pm 0.08^{b}$ & $4.23 \pm 0.14^{a}$ & $4.72 \pm 0.21^{b c}$ & $4.83 \pm 0.26^{b}$ & $4.79 \pm 0.15^{a b}$ & $4.19 \pm 0.18^{a}$ & $3.30 \pm 0.23^{a c}$ \\
\hline \multicolumn{8}{|l|}{ E1 } \\
\hline E2 & $4.18 \pm 0.32^{\mathrm{a}}$ & $4.42 \pm 0.33^{a}$ & $4.97 \pm 0.35^{\mathrm{cd}}$ & $4.12 \pm 0.15^{\mathrm{a}}$ & $4.65 \pm 0.07^{a}$ & $4.44 \pm 0.23^{\mathrm{ac}}$ & $3.72 \pm 0.32^{b c d}$ \\
\hline E3 & $4.10 \pm 0.16^{a}$ & $4.33 \pm 0.18^{a}$ & $4.54 \pm 0.15^{a b}$ & $4.21 \pm 0.31^{a}$ & $4.64 \pm 0.09^{a}$ & $4.93 \pm 0.21^{b d}$ & $3.14 \pm 0.20^{a}$ \\
\hline E4 & $4.03 \pm 0.21^{a}$ & $4.45 \pm 0.23^{a}$ & $4.26 \pm 0.32^{a}$ & $4.83 \pm 0.21^{b c}$ & $4.62 \pm 0.26^{a b}$ & $4.30 \pm 0.21^{\mathrm{ac}}$ & $3.04 \pm 0.18^{a}$ \\
\hline C1 & $4.81 \pm 0.14^{b}$ & $5.18 \pm 0.11^{b}$ & $5.44 \pm 0.11^{\text {ef }}$ & $5.18 \pm 0.08^{b c}$ & $5.18 \pm 0.12^{\mathrm{ac}}$ & $4.62 \pm 0.05^{\mathrm{acd}}$ & $3.58 \pm 0.11^{\mathrm{c}}$ \\
\hline $\mathrm{C} 2$ & $4.76 \pm 0.18^{a b}$ & $5.05 \pm 0.19^{b}$ & $5.73 \pm 0.21^{f}$ & $5.24 \pm 0.18^{\mathrm{C}}$ & $5.11 \pm 0.15^{\mathrm{a}}$ & $4.74 \pm 0.10^{\mathrm{bcd}}$ & $3.74 \pm 0.14^{\mathrm{cd}}$ \\
\hline C3 & $4.27 \pm 0.11^{a}$ & $5.04 \pm 0.09^{b}$ & $5.53 \pm 0.09^{e f}$ & $4.72 \pm 0.06^{b}$ & $4.98 \pm 0.10^{a}$ & $4.63 \pm 0.06^{b c d}$ & $3.96 \pm 0.07^{d}$ \\
\hline C4 & $4.34 \pm 0.12^{a}$ & $5.08 \pm 0.30^{b}$ & $5.11 \pm 0.12^{\mathrm{de}}$ & $4.77 \pm 0.26^{b}$ & $4.73 \pm 0.34^{a}$ & $4.23 \pm 0.31^{\mathrm{ac}}$ & $3.82 \pm 0.31^{\mathrm{cd}}$ \\
\hline \multicolumn{8}{|c|}{ ENTEROBACTERIA } \\
\hline & $2.99 \pm 0.24^{b}$ & $2.33 \pm 0.28^{b}$ & $2.40 \pm 0.18^{\mathrm{cd}}$ & $1.03 \pm 0.11^{a}$ & & $<1$ & $<1$ \\
\hline E1 & & & & & $<1$ & & \\
\hline E2 & $2.24 \pm 0.06^{a}$ & $2.13 \pm 0.14^{b c}$ & $1.09 \pm 0.10^{a}$ & $<1$ & $<1$ & $<1$ & $<1$ \\
\hline E3 & $2.00 \pm 0.13^{a}$ & $2.26 \pm 0.09^{b c}$ & $1.84 \pm 0.16^{\mathrm{C}}$ & $1.32 \pm 0.13^{a}$ & $1.05 \pm 0.21^{a}$ & $<1$ & $<1$ \\
\hline E4 & $2.19 \pm 0.07^{a}$ & $1.74 \pm 0.21^{\mathrm{ac}}$ & $1.45 \pm 0.23^{b}$ & $1.19 \pm 0.21^{a}$ & $1.02 \pm 0.07^{a}$ & $<1$ & $<1$ \\
\hline $\mathrm{C} 1$ & $3.91 \pm 0.09^{c}$ & $4.11 \pm 0.15^{d}$ & $3.53 \pm 0.15^{\mathrm{e}}$ & $2.37 \pm 0.24^{b}$ & $2.32 \pm 0.11^{b}$ & $1.63 \pm 0.13^{b}$ & $1.14 \pm 0.05^{a}$ \\
\hline $\mathrm{C} 2$ & $3.82 \pm 0.13^{\mathrm{C}}$ & $4.02 \pm 0.21^{d g}$ & $3.13 \pm 0.09^{d e}$ & $2.50 \pm 0.18^{b}$ & $2.67 \pm 0.15^{b c}$ & $1.84 \pm 0.21^{b}$ & $1.45 \pm 0.12^{a b}$ \\
\hline C3 & $3.89 \pm 0.18^{C}$ & $3.97 \pm 0.12^{\mathrm{df}}$ & $3.23 \pm 0.18^{\mathrm{e}}$ & $2.15 \pm 0.09^{b}$ & $2.31 \pm 0.13^{b}$ & $1.54 \pm 0.18^{b}$ & $1.55 \pm 0.08^{b}$ \\
\hline $\mathrm{C} 4$ & $3.98 \pm 0.12^{\mathrm{C}}$ & $3.31 \pm 0.23^{\mathrm{de}}$ & $3.64 \pm 0.14^{e}$ & $2.06 \pm 0.09^{b}$ & $2.09 \pm 0.21^{b d}$ & $1.14 \pm 0.13^{a}$ & $1.39 \pm 0.03^{a b}$ \\
\hline
\end{tabular}

a-g for each medium data in the same column with different superscript letters are significantly different $(P<0.05)$. 


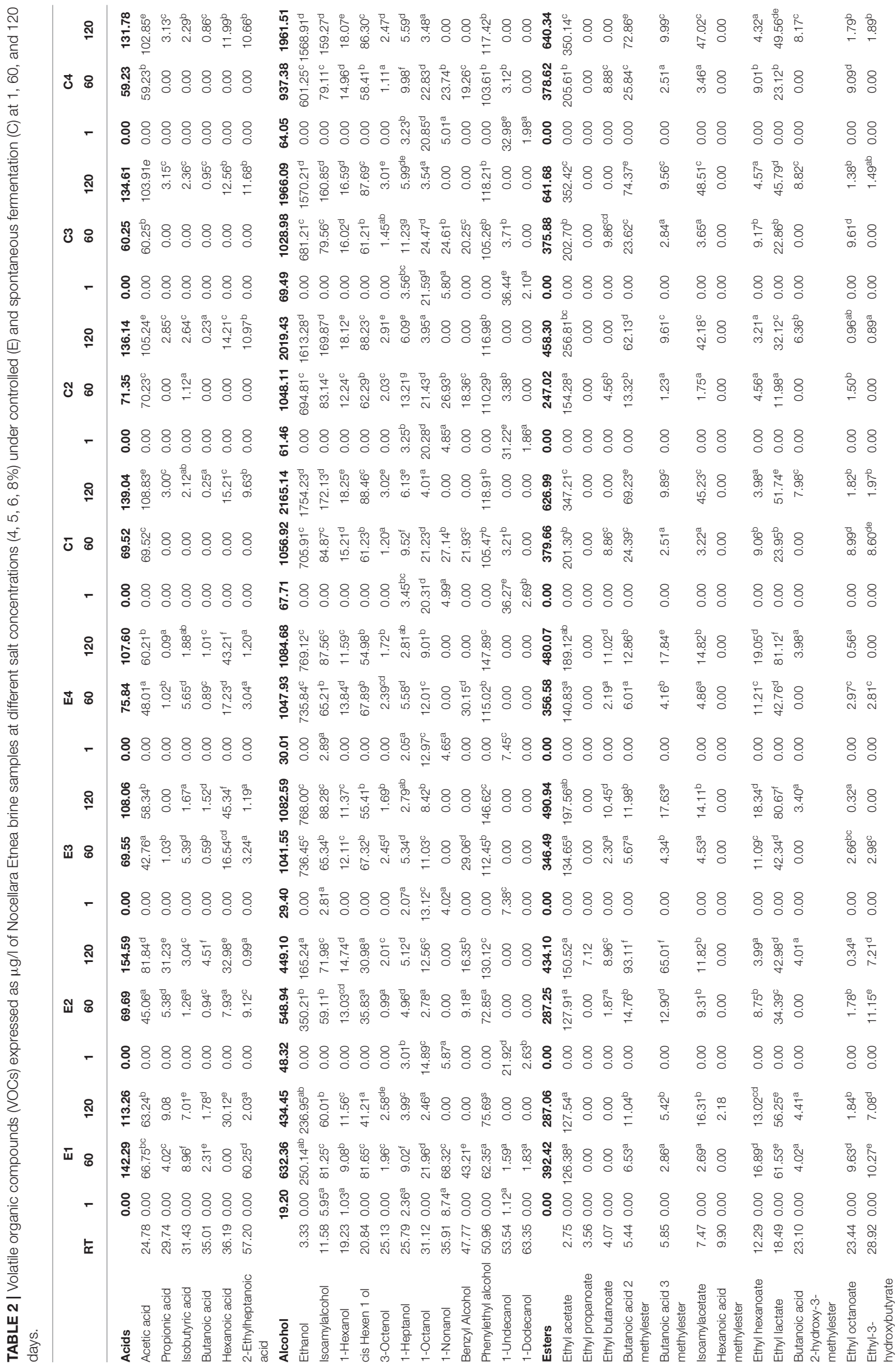




\begin{tabular}{|c|c|c|c|c|c|c|c|c|}
\hline ని & 总 & 爱 & 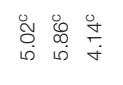 & 总 & 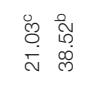 & 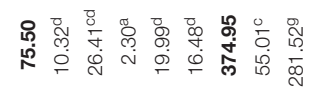 & 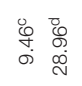 & 离 \\
\hline đ̊ : & $\underset{\substack{0 \\
0}}{0}$ & 8 & 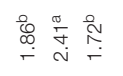 & $\begin{array}{l}\stackrel{\circ}{0} \\
\stackrel{\infty}{\circ}\end{array}$ & 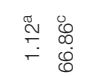 & 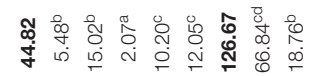 & 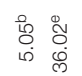 & 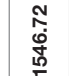 \\
\hline- & $\stackrel{8}{\circ}$ & $\stackrel{8}{\circ}$ & $\begin{array}{lll}8 & 8 & 0 \\
0 & 0 & 0 \\
0\end{array}$ & 8 & $\begin{array}{l}8 \\
8 \\
0\end{array}:$ & 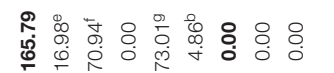 & $\begin{array}{l}8 \\
0 \\
0\end{array}$ & 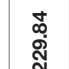 \\
\hline 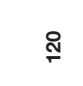 & 尊 & 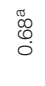 & 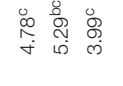 & $\stackrel{\substack{0 \\
\infty}}{+\infty}$ & 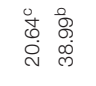 & 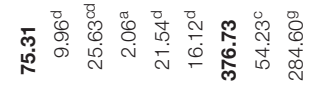 & 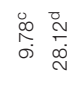 & 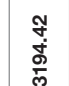 \\
\hline 88 & $\underset{\substack{\infty \\
0}}{\stackrel{0}{0}}$ & $\stackrel{\circ}{\circ}$ & 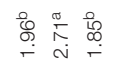 & $\begin{array}{l}0 \\
\stackrel{+}{0} \\
\stackrel{0}{0}\end{array}$ & 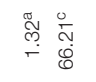 & 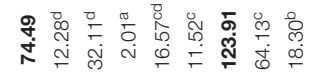 & 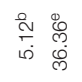 & 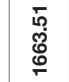 \\
\hline- & $\stackrel{\circ}{\circ}$ & $\stackrel{8}{\circ}$ & 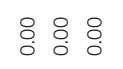 & $\stackrel{8}{\circ}$ & : & 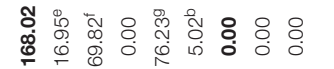 & : & 容 \\
\hline$\stackrel{乛}{\mp}$ & 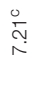 & $\stackrel{8}{\circ}$ & 惌 & $\stackrel{\stackrel{m}{\rho}}{\sim}$ & \begin{tabular}{l}
$:$ \\
\hdashline \\
$\infty$ \\
$\infty$
\end{tabular} & 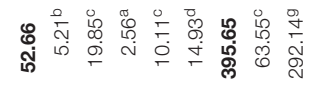 & 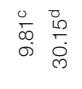 & $\stackrel{\infty}{\bar{j}}$ \\
\hline ชั ๕ & 舀 & $\stackrel{8}{\circ}$ & 总 & : & 总哭 & 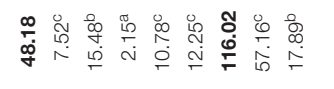 & 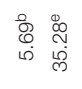 & 兽 \\
\hline- & $\stackrel{\circ}{\circ}$ & $\stackrel{8}{\circ}$ & : : : : : & $\stackrel{8}{0}$ & : : & 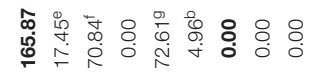 & $: 0$ & 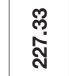 \\
\hline$\stackrel{乛}{\leftarrow}$ & 兽 & 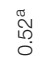 & 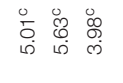 & $\underset{\substack{0 \\
\infty}}{+\infty}$ & 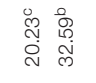 & 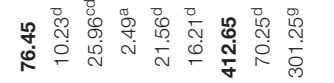 & 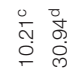 & సิ̃ \\
\hline j 8 & $\begin{array}{c}\stackrel{\circ}{\alpha} \\
\text { ò } \\
\dot{0}\end{array}$ & $\stackrel{\circ}{\circ}$ & 亭 & $\begin{array}{l}0 \\
: \\
:\end{array}$ & 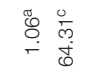 & $\begin{array}{ll}0 \\
0\end{array}$ & $\begin{array}{ll}0 \\
0 \\
0 \\
0 \\
0 \\
0 \\
0\end{array}$ & 产 \\
\hline- & $\stackrel{0}{\circ}$ & $\stackrel{\circ}{\circ}$ & : : : : & $\stackrel{8}{8}$ & : & 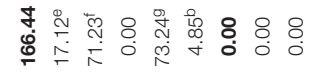 & $8:$ & 点 \\
\hline$\stackrel{乛}{\check{1}}$ & 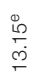 & $\stackrel{\circ}{\circ}$ & & 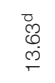 & & 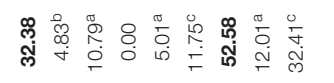 & 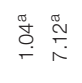 & 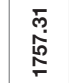 \\
\hline İ \& & 商 & $\stackrel{8}{0}$ & 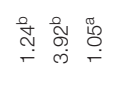 & $\begin{array}{l}\stackrel{\leftrightarrow}{\circ} \\
\stackrel{0}{0} \\
\stackrel{0}{0}\end{array}$ & 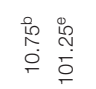 & 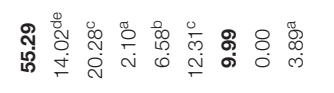 & 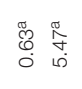 & 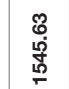 \\
\hline- & $\stackrel{\circ}{\circ}$ & $\stackrel{8}{\circ}$ & : & : & 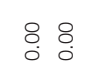 & 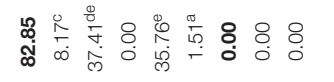 & : & 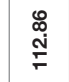 \\
\hline$\stackrel{乛}{\leftarrow}$ & 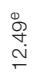 & $\stackrel{8}{\circ}$ & 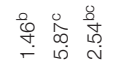 & 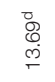 & & 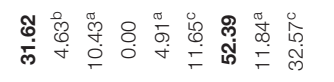 & 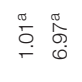 & 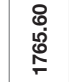 \\
\hline జూ \& & 总 & $\stackrel{8}{\circ}$ & 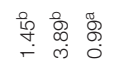 & $\begin{array}{l}\stackrel{0}{0} \\
\stackrel{0}{0} \\
\stackrel{0}{0}\end{array}$ & 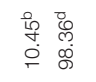 & 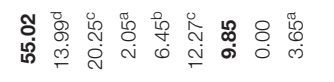 & 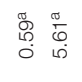 & 胥 \\
\hline- & $\stackrel{8}{0}$ & 8 & : & : & : & 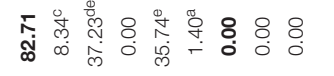 & : & 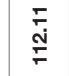 \\
\hline$\stackrel{2}{\stackrel{2}{+}}$ & 总 & $\stackrel{8}{0}$ & 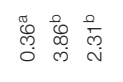 & $\stackrel{8}{\stackrel{8}{\circ}}$ & & 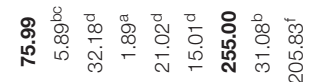 & 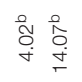 & 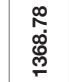 \\
\hline พิ : & $\stackrel{8}{\circ}$ & $\stackrel{8}{\circ}$ & 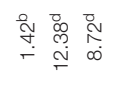 & 8 & 赵 & 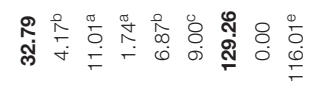 & 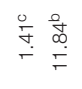 & 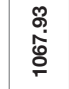 \\
\hline- & $\stackrel{\circ}{\circ}$ & $\stackrel{8}{\circ}$ & : : : : & 8 & 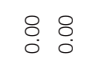 & 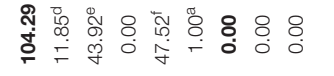 & $\circ:$ & 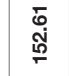 \\
\hline$\stackrel{1}{\stackrel{2}{9}}$ & $\stackrel{\stackrel{0}{N}}{N}$ & : & 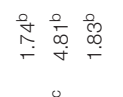 & 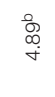 & & 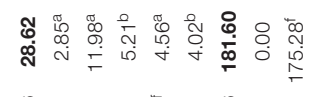 & 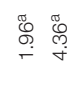 & 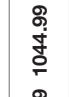 \\
\hline$\overline{4}:$ & 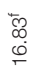 & $\stackrel{8}{\circ}$ & 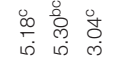 & 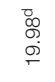 & 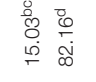 & 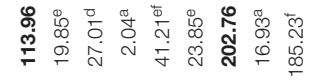 & 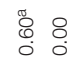 & 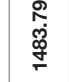 \\
\hline- & $\stackrel{8}{\circ}$ & $\stackrel{8}{\circ}$ & $\begin{array}{lll}8 & 0 \\
0 & 0 & 0 \\
0 & 0\end{array}$ & : & : 8 & 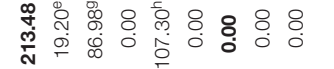 & $\begin{array}{ll}8 \\
0 \\
0 & 0 \\
0\end{array}$ & 品 \\
\hline$\underline{\underline{x}}$ & 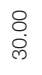 & 总 & 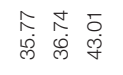 & $\begin{array}{l}\bar{\sigma} \\
\text { bु }\end{array}$ & 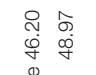 & 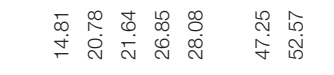 & $\left.\begin{array}{ll}0 & 0 \\
0 \\
0 \\
0\end{array}\right]$ & \\
\hline
\end{tabular}



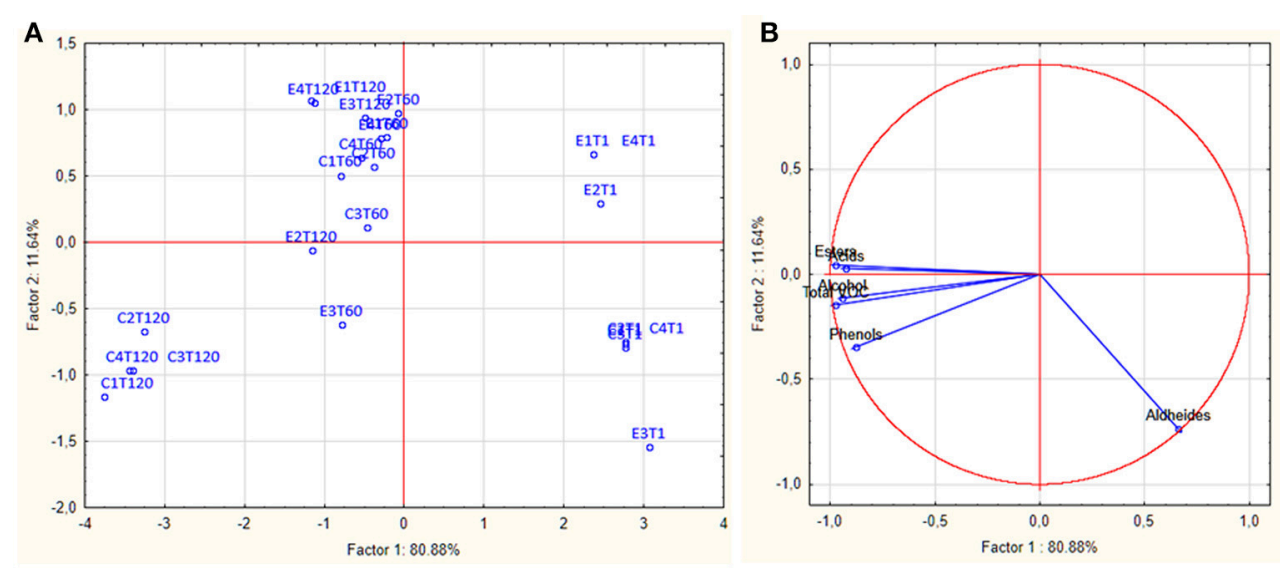

FIGURE 3 | PCA plot (A) and score plot (B) showing the distribution of experimental (E) and control (C) samples through the fermentation process.

was mainly represented by aldehydes. Score plots are effective in showing the difference among samples and in separating them in the graphs. In detail, all E and C samples at 1 day of fermentation highlighted a positive contribution by component 2 planes. After $120 \mathrm{~d}, \mathrm{C}$ samples were characterized by a positive contribution of alcohols whereas E samples principally by esters.

\section{Permutation Analysis and Correlations Between Microbiota and Metabolome of Nocellara Etnea Table Olives}

Similarities in the observed microbial counts and metabolomics profiles between samples at 60 and 120 days of fermentation were estimated using the PermutMatrixEN software (Figure 4). In detail, two clusters were revealed, showing that overall the samples grouped based on the addition of starters and on time of the fermentation. It is interesting to note that salt content did not discriminate samples, with the exception of the experimental samples E1 and E2 at 60 and 120 d, respectively, which exhibited unique profiles. In fact, these samples showed the most divergent microbial and metabolic profiles, with a strong presence of ethyl-3-hydroxybutyrate, LAB, mesophilic bacteria, isobutyric acid, 2-ethyleptanoic acid, 1-dodecanol, decanoic acid methyl ester, methyl idrocynnamate, 1-octanol, 1-nonanol, ethyl octanoate, benzyl alcohol, staphylococci, octanal, decanal, benzaldehyde, nonanal in the E1 sample at 60 days, and of butanoic acid 2 methylester, propionic acid, ethyl propanoate, butanoic acid 3 methylester, hexanoic acid, phenylethyl alcohol, ethyl butanoate in the E2 sample at 120 days. Compared to 60 days of fermentation, control samples at 120 days showed different microbial and metabolomics profiles with a strong increase in acetic acid, phenol, ethanol, isoamyl alcohol, ethyl acetate, 1-hexanol, isoamylacetate, butanoic acid 2-hydroxy-3-methylester, creosol (homoguaiacol), 3-octenol, cis hexen $1 \mathrm{ol}$, ethyl decanoate, ethyl dodecanoate, and nonanal. Evaluating E samples, both, time of fermentation and $\mathrm{NaCl}$ content, affected the microbial and metabolomics profiles. High similarity was found between samples treated with
6 and $8 \%$ of $\mathrm{NaCl}$ after 60 and $120 \mathrm{~d}$ of fermentation, characterized by a lower amount of guaiacol, 4-ethyl phenol and enterobacteria.

Correlations between microbial and metabolomics data are shown in Figure 5. Among organic acids, propionic, isobutyric acid, butanoic, and exanoic compounds were negatively correlated with yeasts and staphylococci, and positively correlated $(r>0.30, p<0.05)$ with mesophilic bacteria. One exception was the acetic acid, which, was positively correlated $(r=0.238, p=0.045)$ with enterobacteria and negatively with LAB $(r=-0.735 ; p=0.048)$. Zooming on the metabolomics, the acetic acid was positively correlated $(r>0.70, p<0.05)$ with alcohols (ethanol, isoamylalcohol,1-hexanol, cis hexen $1 \mathrm{ol}$ and 3-octenol) and esters (ethyl acetate, butanoic acid 2 methylester, isoamylacetate, butanoic acid, and 2-hydroxy-3-methylester). Alcohols, with the exception of 3-octenol, phenylethyl alcohol and 1-dodecanol, were positively correlated with enterobacteria and yeasts. In addition, ethanol, isoamylalcohol, 1-hexanol, cis hexen $1 \mathrm{ol}$, and 3 -octenol were negatively correlated with mesophilic bacteria and LAB.

\section{Molecular Identification of Lactobacillus spp. Isolates}

Four hundred isolates from MRS plates were considered lactobacilli based on their positive Gram reaction, non-motility, absence of catalase activity, and spore formation, and rod or coccal shape. Presumptive lactobacilli were identified by using multiplex PCR and were ascribed to L. plantarum, L. pentosus, L. paracasei, and Lactobacillus casei species, and their occurrence percentage in $\mathrm{E}$ and $\mathrm{C}$ samples, at 1, 60, and 120 days of fermentation is illustrated in Figure 6. In detail, $\mathrm{C}$ samples, as expected, exhibited at both day 1 and after 60 days, a high occurrence $(70 \%)$ of $L$. plantarum accompanied by L. pentosus (30\%). After $120 \mathrm{~d}$, a slight occurrence (20\%) of L. casei was detected. In E samples, $40 \%$ of isolates were ascribed to both L. paracasei and L. plantarum. The remaining $20 \%$ was identified as $L$. pentosus. Similar occurrence was revealed after $60 \mathrm{~d}$ of fermentation. Different species occurrence was highlighted at 


\section{Dissimilarity : - Euclidean distance}

\section{The colors scale:}

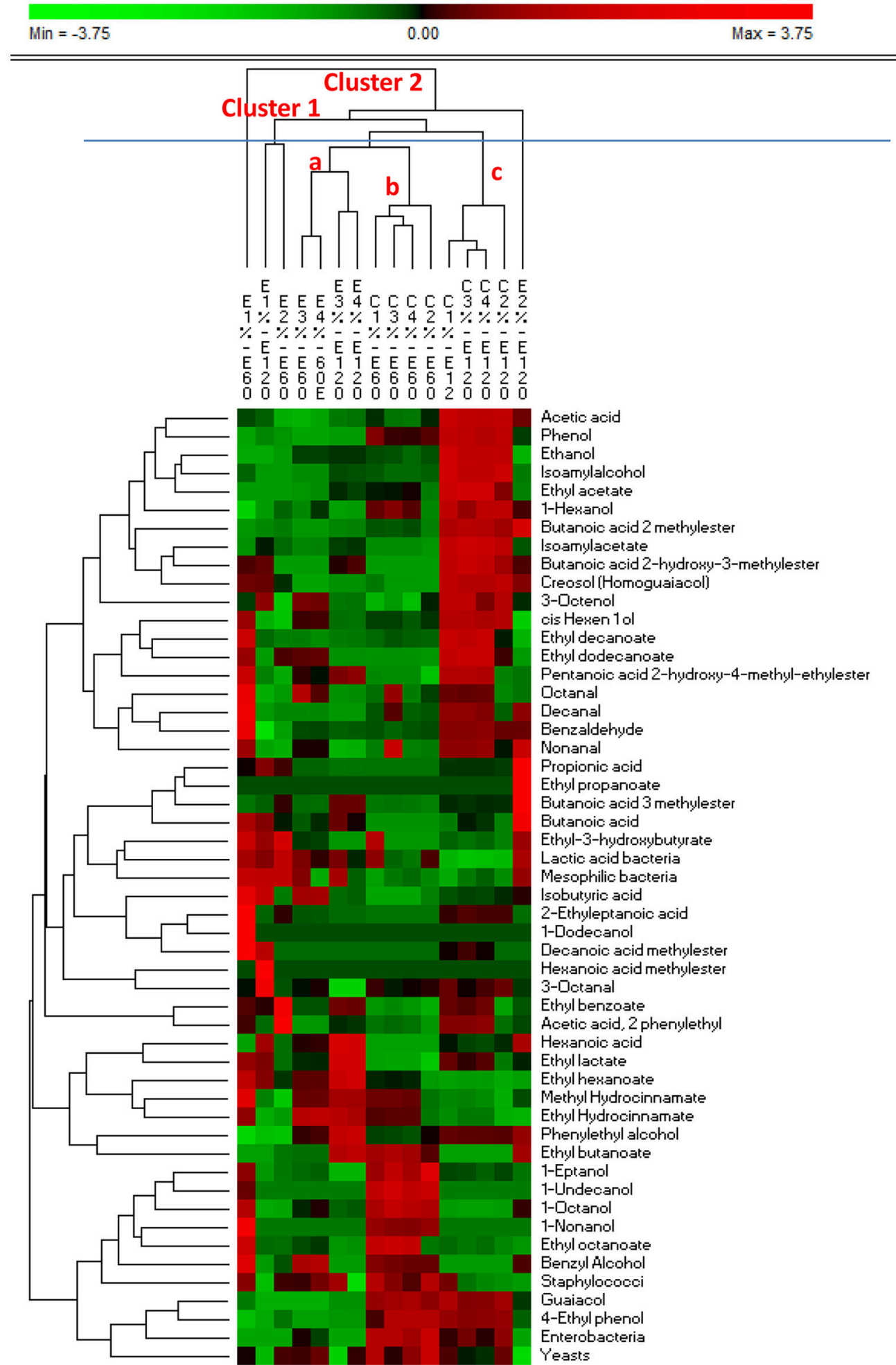

FIGURE 4 | PermutMatrixEN analysis of the microbial and metabolomics profiles of experimental (E) and control (C) Nocellara Etnea under different salt concentrations $(4,5,6,8 \%)$ at 60 and 120 days. 


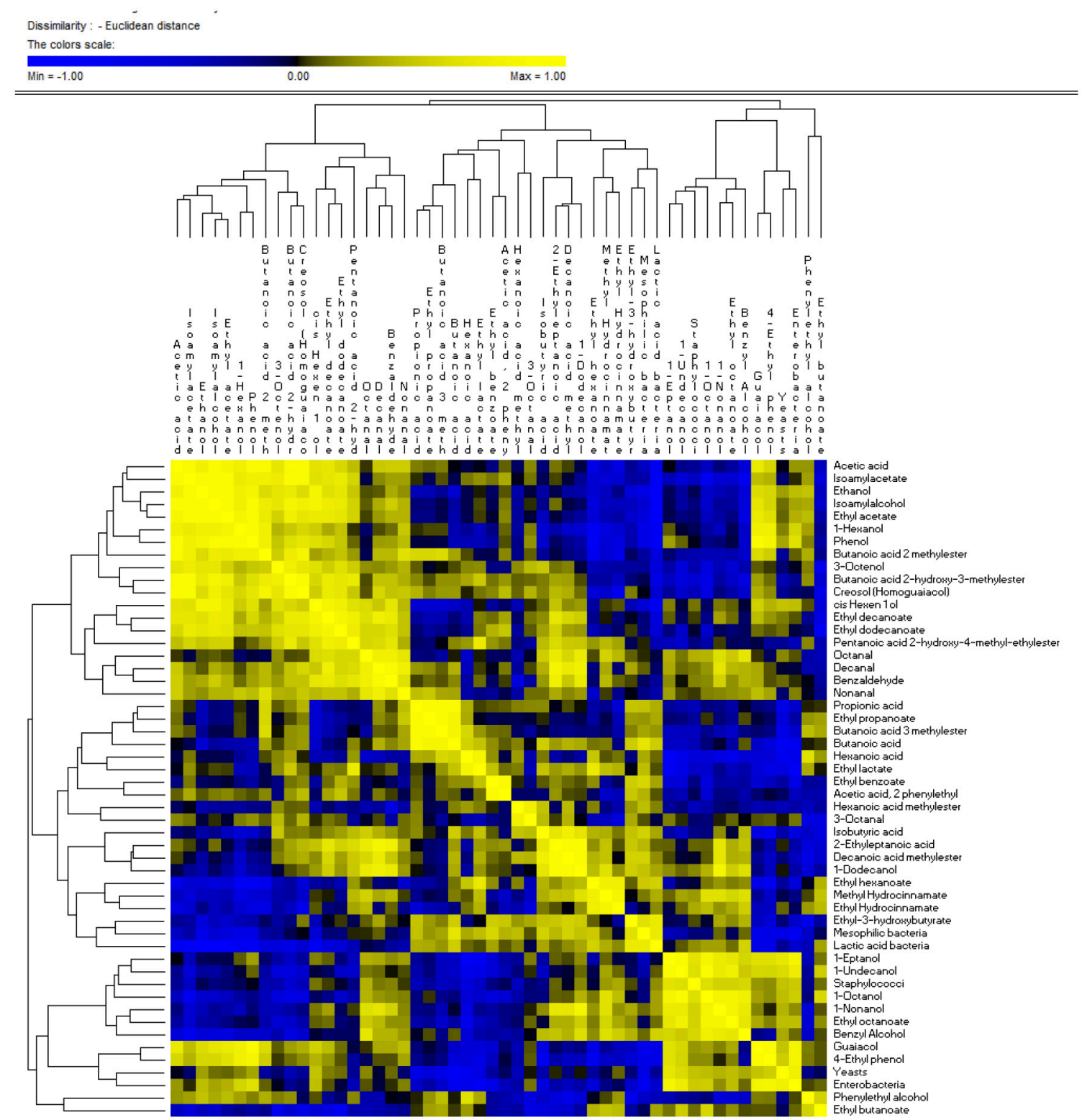

FIGURE 5 | Significant correlations between microbial and metabolomics data found after 60 and 120 days of fermentation of experimental (E) and control (C) Nocellara Etnea under different salt concentrations (4, 5, 6, 8\%).

120 days, since the majority of the isolates were identified as $L$. paracasei (60\%), followed by $L$. pentosus $(30 \%)$ and L. plantarum (10\%) (Figure 6). It is interesting to note that among the 60 isolates identified as $L$. paracasei, the majority of the occurrence was revealed in E sample at $5 \%$ of salt (E2).

\section{Viability of $L$. paracasei N24 Strain Through Table Olives Fermentation}

In order to evaluate the viability of $L$. paracasei strain N24 in the experimental table olives at 60 and $120 \mathrm{~d}$, DNA of 60 strains was submitted to REP-PCR. Results are reported in Figures 7A,B. All strains clustered together with the L. paracasei
N24 strain (with a percentage of similarity higher than $85 \%$ ), with the exception of 17 strains, which exhibited different profiles.

\section{Sensory Data}

Results of sensory analysis are reported in Table 3. No offodors were detected in any samples as inferred by the low scores of the taste panel for this organoleptic perception. Overall, regarding the gustatory sensations (acidity, saltiness, and bitterness), differences among $\mathrm{E}$ and $\mathrm{C}$ samples at different salt content were detected, with the exception of bitterness descriptor. In detail, E samples received similar and moderate values in 


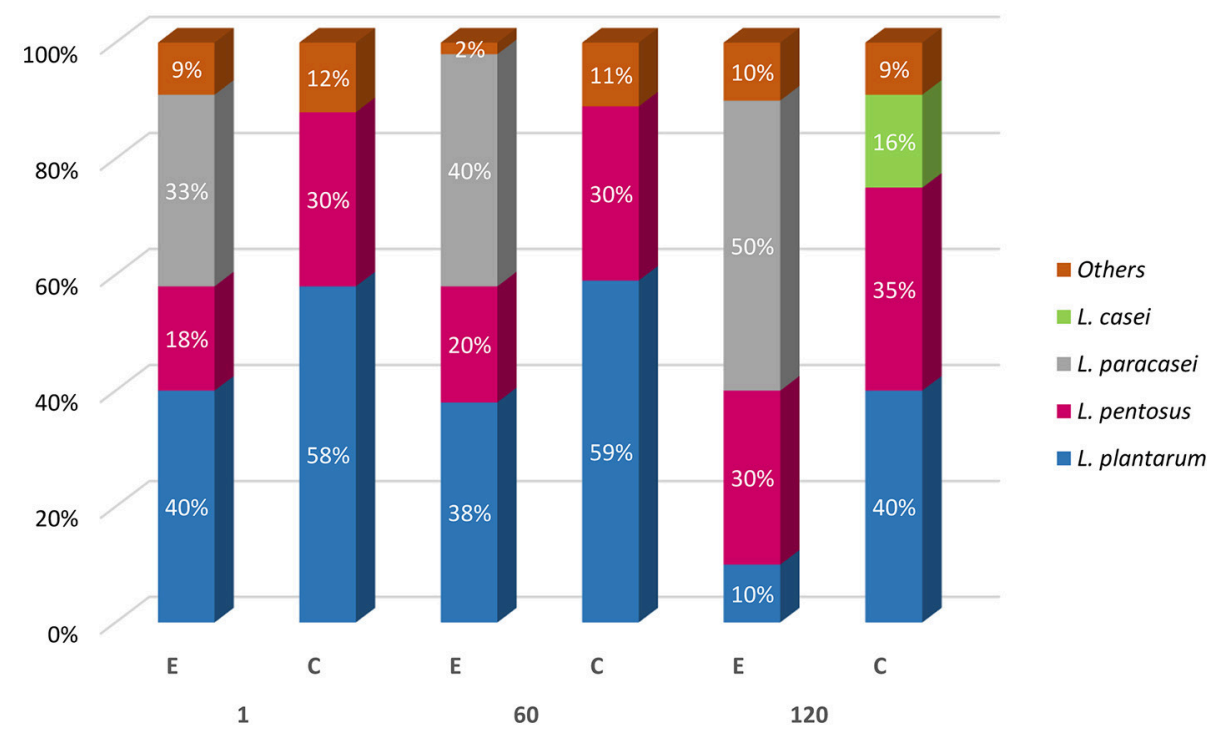

FIGURE 6 | Molecular identification of Lactobacillus spp. isolates from experimental (E) and control (C) samples at 1, 60, and 120 days of fermentation.

acidity, while $\mathrm{C}$ ones exhibited a higher score, with the highest value for the sample with $8 \%$ of salt (C4). A similar trend was observed for the saltiness score, with lower value in E samples. Only samples made adding $8 \%$ of salt (E4 and C4) showed significant differences, registering a saltiness score of about 8.1. Regarding kinaesthetic sensations (hardness, fibrousness, and crunchiness) no statistical differences were achieved among samples, registering a mean score of 7.2. Finally, E olives received higher scores for the overall acceptability descriptor, with the exception of E1 sample, which showed an average score similar to those obtained by $\mathrm{C}$ samples. Experimental E2 sample exhibited the highest overall acceptability score $(8.8 \pm$ $0.82)$.

\section{DISCUSSION}

Consumer's acceptance and attitude toward functional foods determine the market success, which is growing steadily, mainly toward vegetables, fruits, and cereal products due to vegetarianism emergence, lactose intolerance, cholesterolemia, and food allergies (Granato et al., 2010; Ranadheera et al., 2010; Peres et al., 2012; Martins et al., 2013). Among the non-dairy functional foods, table olives represent a good food matrix to carry active viable bacteria into the gastrointestinal tract (Lavermicocca et al., 2005; Valerio et al., 2006; Abriouel et al., 2012; Randazzo et al., 2017). Table olives are considered functional food because of their nutritional value related to the presence of phenolic compounds and monounsaturated fatty acids (Buckland and Gonzalez, 2015). Nevertheless, their preparation relies on the use of $\mathrm{NaCl}$ as the main ingredient of the brine especially for reducing undesirable spoilage and pathogenic microorganisms ensuring, thus, the microbiological safety and quality of the final product (Taormina, 2010; Albarracín et al., 2011). In recent years, public health and regulatory authorities have recommended the reduction of dietary intake of sodium because of its association to hypertension [World Health Organisation (WHO), 2003, 2007], and to cardiovascular diseases (Ortega et al., 2011). In Mediterranean regions, populations eat considerably high amounts of table olives and, subsequently, ingest greater amounts of salt; hence, $\mathrm{NaCl}$ reduction in table olives is strongly recommended. Recently, several studies have been focused on the replacement of $\mathrm{NaCl}$ with other salts, such as $\mathrm{KCl}$ and $\mathrm{CaCl}_{2}$ (Bautista-Gallego et al., 2010, 2013a,b; Mateus et al., 2016; Zinno et al., 2017), and results are not fully in areement. In particular, while Bautista-Gallego et al. (2010) showed that Enterobacteriaceae growth was slightly stimulated by high $\mathrm{CaCl}_{2}$ contents, Mateus et al. (2016) revealed that the presence of potassium and calcium chlorides in the brines caused an increase of the enterobacteria death rate. Up to now only De Bellis et al. (2010) have proposed to study table olive processing at reduced $\mathrm{NaCl}$ concentration, without any salt replacement. Based on our previous reported data (Randazzo et al., 2017), with the aim to set up functional table olives from Nocellara Etnea cultivar, in the present study the fermentation was carried out at different salt contents $(4,5,6$, and $8 \%)$, by using starter cultures constituting of the promising probiotic strain $L$. paracasei $\mathrm{N} 24$ and by the strain L. plantarum UT2.1. The fermentation without the use of starter cultures was used as control. It is well established that both salt content and $\mathrm{pH}$ value are the main parameters controlling the pathogens growth in fermented products, such as C. botulinum. Taormina (2010) has already reported that the probability of growth and toxin production of C. botulinum at $5 \% \mathrm{NaCl}$ decreased as the $\mathrm{pH}$ and storage temperature was decreased. In this context, our data reveled that all experimental brines have had a $\mathrm{pH}$ value below 4.5, with the exception of control sample $\mathrm{C} 4$, and a constant temperature of ca $18^{\circ} \mathrm{C}$, which guarantee the pathogens growth inhibition. Overall, in contrast to De Bellis et al. (2010), who observed 
A

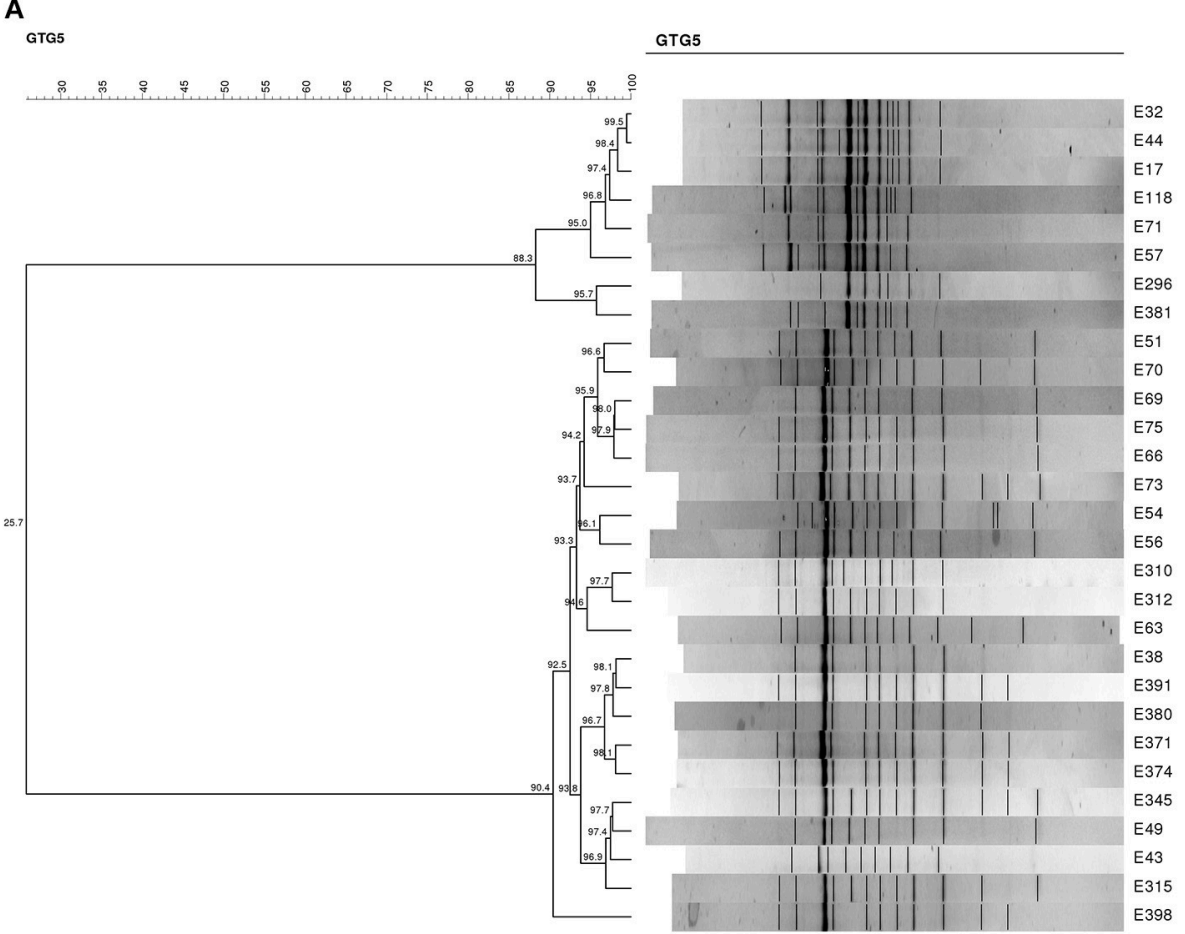

B

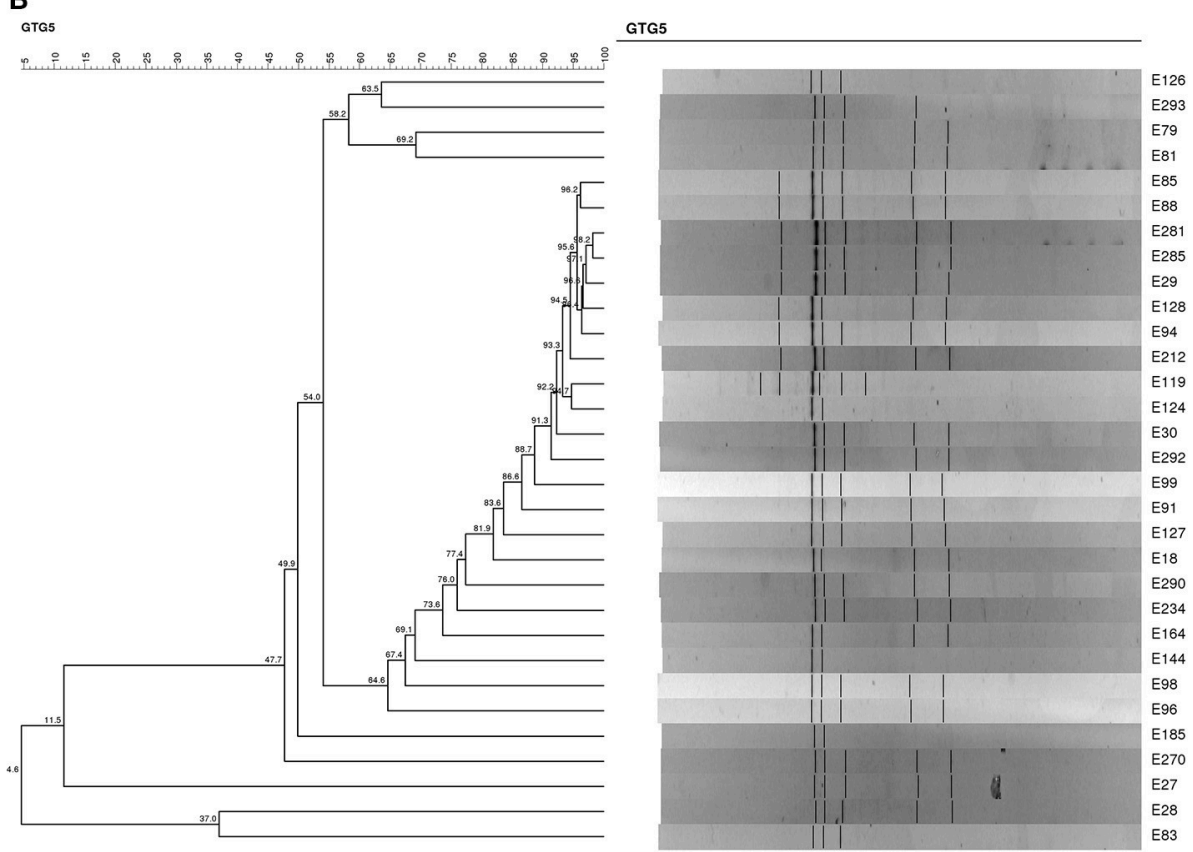

FIGURE 7 | (A) Dendrogram generated after cluster analysis of the digitized (GTG) 5 -PCR fingerprints of the lactobacilli isolated from experimental table olives at 60 and 120 days. (B). Dendrogram generated after cluster analysis of the digitized (GTG) $5-P C R$ fingerprints of the lactobacilli isolated from experimental table olives at 60 and 120 days.

alterative processes in spontaneous fermentation at $4 \%$ of $\mathrm{NaCl}$, in the present study, samples treated with different salt content obtained similar scores in terms of "abnormal fermentation" sensory attribute. Slight sensory differences were detected only for bitterness and acidity descriptors among experimental and control samples, with a higher score in control ones. These 


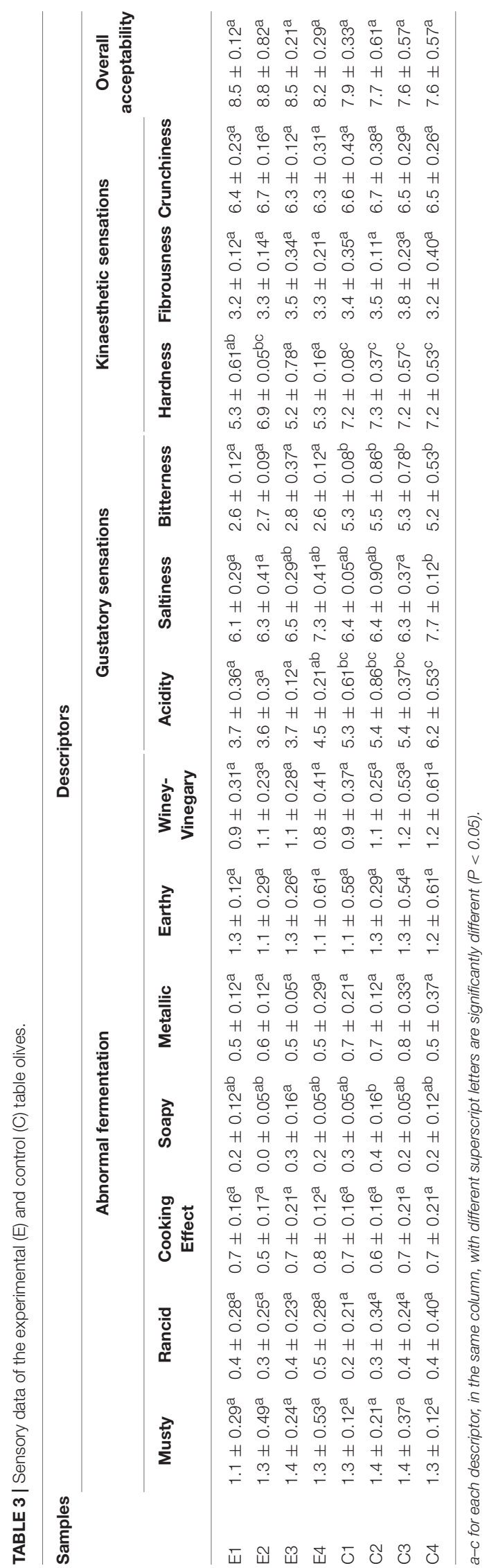

differences could be therefore attributed to the added starter cultures. In particular, the higher contents of acetic acid, ethanol, and phenols, associated to vinegary, fatty smell, and bitter aroma, respectively, in control samples, could justify the highest acidity and bitterness scores registered by panelists. On the contrary, it is interesting to note that the experimental sample at $5 \%$ of $\mathrm{NaCl}$ (E2) showed the highest overall acceptability score and, based on the similarity tree, this sample exhibited a unique profile. Even in the experimental samples a high amount of propionic acid was detected, panelists did not reveal any offflavors, indicating that the defect generated by propionic acid was probably hidden by other compounds such as esters, as indicated by Blana et al. (2014). In fact, among total VOCs, esters showed an occurrence percentage higher in experimental samples rather than in control samples, contributing to more pleasant flavors (Sabatini et al., 2008). Evaluating both PCA data on VOCs and similarity tree, brine samples were mainly grouped based on fermentation time, in discordance to Blana et al. (2014), that demonstrated the importance of salt content on the fermentation profiles. The present study revealed that salt contents slightly influences the metabolome of table olives; however, the overall characteristics of the final products were strongly time-dependent. In addition, the salt content did not affect the performances of $L$. paracasei N24 and L. plantarum UT2.1 starter cultures used. They were effective in accelerating the fermentation process, quickly reducing the $\mathrm{pH}$ from the 7th day of fermentation, inhibiting spoilage bacteria in all experimental samples. In fact, at the end of the fermentation, Enterobacteriaceae were countable only in control samples. This microbial group, as suggested by Medina-Pradas et al. (2017), can negatively influence the quality and safety of table olives, causing gas pockets spoilage, or producing metabolites that affect the final aroma. Moreover, the present work allowed to asses that starters, rather than $\mathrm{NaCl}$ replacement with $\mathrm{CaCl}_{2}$ and/or $\mathrm{KCl}$, as discussed by Mateus et al. (2016), has an effect on the reduction of the yeast population. In fact, in the present study yeast decreasing could be attributed to the intense competition between LAB and yeasts for nutrients. It is noteworthy that yeasts are involved in the VOCs formation; nevertheless, a high occurrence of this microbial group could be responsible of undesirable fermentation. In the present study, yeasts were positively correlated with the main alcohols and phenols detected, which could generate off-flavors. Hence, the use of starter cultures is strongly recommended in table olives fermentation also in order to inhibit spoilage bacteria and control the autochthonous yeast growth. Evaluating the lactobacilli behavior, L. plantarum and $L$. pentosus were the main species detected at the end of the fermentation in all samples, confirming their key role in table olive fermentation. In addition, a high survival rate of the promising probiotic N24 strain was depicted in all experimental samples. This evidence confirms its technological suitability to be used as starter in olive fermentations as well as its ability to survive during the process regardless of brine salt concentration. In addition, the promising probiotic L. paracasei N24 strain exhibited the highest occurrence in experimental sample at 5\% of salt (E2). The latter was clearly separated from the remaining treatments, exhibiting a unique metabolomics profile, which 
generate sensorial traits appreciate by panelists. Hence, data of the present investigation revealed promising perspectives for the application of $L$. paracasei N24 strain as starter cultures for the production of table olives with increased added value.

\section{CONCLUSION}

Results of the present study demonstrated that both brine microbial population and VOCs were slightly affected by salt content while a strong influence was determined by time of fermentation. The reduction of $\mathrm{NaCl}$ content, without any replacement with other salts resulted in a successful fermentation of Nocellara Etnea table olives. The final products fulfilled microbiological criteria and exhibited more appreciate sensorial characteristics. In addition, the formulation of probiotic table olives with low salt content is healthier and more suitable for consumers at risk of hypertension, opening new perspectives for their production at industrial scale.

\section{REFERENCES}

Abriouel, H., Benomar, N., Cobo, A., Caballero, N., Fernández Fuentes, M. Á., Pérez-Pulido, R., et al. (2012). Characterization of lactic acid bacteria from naturally-fermented Manzanilla Aloreña green table olives. Food Microbiol. 32, 308-316. doi: 10.1016/j.fm.2012.07.006

Albarracín, W., Sánchez, I. C., Grau, R., and Barat, J. M. (2011). Salt in food processing; usage and reduction: a review. Int. J. Food Sci. Technol. 46, 1329-1336. doi: 10.1111/j.1365-2621.2010.02492.x

Arroyo-López, F. N., Bautista-Gallego, J., Domínguez-Manzano, J., RomeroGil, V., Rodriguez-Gómez, F., García-García, P., et al. (2012). Formation of lactic acid bacteria-yeasts communities on the olive surface during Spanish-style Manzanilla fermentations. Food Microbiol. 32, 295-301. doi: 10.1016/j.fm.2012.07.003

Bautista-Gallego, J., Arroyo-López, F. N., Durán-Quintana, M. C., and GarridoFernández, A. (2010). Fermentation profiles of Manzanilla-Aloreña cracked green table olives in different chloride salt mixture. Food Microbiol. 27, 403-412. doi: 10.1016/j.fm.2009.11.015

Bautista-Gallego, J., Arroyo- López, F. N., López - López, A., and GarridoFernández, A. (2011). Effect of chloride salt mixtures on selected attributes and mineral content of fermented cracked Aloreña olives. Food Sci. Technol. 44, 120-129. doi: 10.1016/j.lwt.2010.06.027

Bautista-Gallego, J., Arroyo-López, F. N., Rantsiou, K., Jiménez-Díaz, R., GarridoFernández, A., and Cocolin, L. (2013a). Screening of lactic acid bacteria isolated from fermented table olives with probiotic potential. Food Res. Int. 50, 135-142. doi: 10.1016/j.foodres.2012.10.004

Bautista-Gallego, J., Arroyo-López, F. N., Romero-Gil, V., Rodríguez-Gómez, F., García-García, P., and Garrido-Fernández, A. (2015). Fermentation profile of green Spanish-style Manzanilla olives according to $\mathrm{NaCl}$ content in brine. Food Microbiol. 49, 56-64. doi: 10.1016/j.fm.2015.01.012

Bautista-Gallego, J., Rantsiou, K., Garrido-Fernández, A., Cocolin, L., and ArroyoLópez, F. N. (2013b). Salt reduction in vegetable fermentation: reality or desire? J. Food. Sci. 78, 1095-1100. doi: 10.1111/1750-3841.12170

Blana, V. A., Grounta, A., Tassou, C. C., Nychas, G. J., and Panagou, E. Z. (2014). Inoculated fermentation of green olives with potential probiotic Lactobacillus pentosus and Lactobacillus plantarum starter cultures isolated from industrially fermented olives. Food Microbiol. 38, 208-218. doi: 10.1016/j.fm. 2013.09.007

Buckland, G., and Gonzalez, C. A. (2015). The role of olive oil in disease prevention: a focus on the recent epidemiological evidence from cohort studies and dietary intervention trials. Br. J. Nutr. 113, S94-S101, doi: $10.1017 /$ S0007114514003936

\section{AUTHOR CONTRIBUTIONS}

AP, AT, and KV performed the experiments, and analyzed data. $\mathrm{AP}$ and $\mathrm{CR}$ wrote the manuscript. $\mathrm{MD}, \mathrm{CR}$, and CC designed the study and contributed to data interpretation. AT and KV co-wrote the manuscript.

\section{ACKNOWLEDGMENTS}

The authors thank Consoli srl Company for kindly provided Nocellara Etnea olives.

\section{SUPPLEMENTARY MATERIAL}

The Supplementary Material for this article can be found online at: https://www.frontiersin.org/articles/10.3389/fmicb. 2018.01125/full\#supplementary-material

Figure S1 | Total phenolic content (mg/l) of control (C) experimental (E) brine samples at 60 and 120 days of fermentation.

Cocolin, L., Alessandria, V., Botta, C., Gorra, R., De Filippis, F., Ercolini, D., et al. (2013). $\mathrm{NaOH}$-debittering induces changes in bacterial ecology during table olives fermentation. PLoS ONE 8:e69074. doi: 10.1371/journal.pone.0069074

De Bellis, P., Valerio, F., Sisto, A., Lonigro, S. L., and Lavermicocca, P. (2010). Probiotic table olives: microbial populations adhering on olive surface in fermentation sets inoculated with the probiotic strain Lactobacillus paracasei IMPC2.1 in an industrial plant. Int. J. Food Microbiol. 140, 6-13. doi: 10.1016/j.ijfoodmicro

European Council (2010). Council Conclusions of 8 June on Action to Reduce Population Salt Intake for Better Health. European Council. Union. 11.11.2010 C305/3-C305/5.

G-Alegria, E., Lopez, I., Ruiz, J. I., Sáenz, J., and Fernández, E., Zarazaga, M., et al. (2004). High tolerance of wild Lactobacillus plantarum and Oenococcus oeni strains to lyophilisation and stress environmental condition of acid $\mathrm{pH}$ and ethanol. FEMS Microbiol. Lett. 230, 53-61. doi: 10.1016/S0378-1097(03)00854-1

Gevers, D., Huys, G., and Swings, J. (2001). Applicability of rep-PCR fingerprinting for identification of Lactobacillus species. FEMS Microbiol. Lett. 205, 31-36. doi: 10.1111/j.1574-6968.2001.tb10921.x

Granato, D., Branco, G. F., Nazzaro, F., Cruz, A. G., and Faria, J. A. F. (2010). Functional foods and nondairy probiotic food development: trends, concepts, and products. Comp. Rev. Food Sci. Food Safety 9, 292-302. doi: $10.1111 / j .1541-4337.2010 .00110 . x$

Hurtado, A., Reguant, C., Bordons, A., and Rozès, N. (2012). Lactic acid bacteria from fermented table olives. Food Microbiol. 31, 1-8. doi: $10.1016 /$ j.fm.2012.01.006

International Olive Council (IOC) (2011). Sensory Analysis of Table Olives. Document COI/OT/MO No 1/Rev. 2, Madrid.

International Olive Council (IOC) (2016). Market Newsletter No 108 - September 2016. Available Online at: http://www.internationaloliveoil.org/news/view/ 686-year2016-news/762-marketnewsletter-september-2016

Lavermicocca, P., Valerio, F., Lonigro, S. L., De Angelis, M., Morelli, L., Callegari, M. L., et al. (2005). Adhesion and survival of lactobacilli and bifidobacteria on table olives with the aim of formulating a new probiotic food. Appl. Environ. Microbiol. 71, 4233-4240. doi: 10.1128/AEM.71.8.42334240.2005

Malheiro, R., de Pinho, P. G., Casal, S., Bento, A., and Pereira, J. A. (2011). Determination of the volatile profile of stoned table olives from different varieties by using HS-SPME and GC/IT-MS. J. Sci. Food Agric. 91, 1693-1701. doi: $10.1002 /$ jsfa. 4372

Martins, E. M. F., Mota Ramos, A., Silva Lago Vanzela, E., César Stringheta, P., de Oliveira Pinto, C. L., and Martins, J. M. (2013). Products of vegetable origin: 
a new alternative for the consumption of probiotic bacteria. Food Res. Int. 51, 764-770. doi: 10.1016/j.foodres.2013.01.047

Mateus, T., Santo, D., Saúde, C., Pires-Cabral, P., and Quintas, C. (2016). The effect of $\mathrm{NaCl}$ reduction in the microbiological quality of cracked green table olives of the Maçanilha Algarvia cultivar. Int. J. Food Microbiol. 218, 57-65. doi: 10.1016/j.ijfoodmicro.2015.11.008

Medina-Pradas, E., Pérez-Díaz, I. M., Garrido-Fernández, A., and ArroyoLópez, F. (2017). "Review of vegetable fermentations with particular emphasis on processing modifications, microbial ecology, and spoilage," in The Microbiological Quality of Food Foodborne Spoilers, eds A. Bevilacqua, M. R. Corbo, and M. Sinigaglia (New York, NY: Elsevier), 211-236.

Ortega, R. M., López Sobaler, A. M., Ballesteros, J. M., Pérez Farinós, N., Rodríguez Rodríguez, E., Aparicio, A., et al. (2011). Estimation of salt intake by $24 \mathrm{~h}$ urinary sodium excretion in a representative sample of Spanish adults. Br. J. Nutr. 105, 787-794. doi: 10.1017/S000711451000423X

Panagou, E. Z., and Tassou, C. C. (2006). Changes in volatile compounds and related biochemical profile during controlled fermentation of $\mathrm{cv}$. Conservolea green olives. Food Microbiol. 23, 738-746. doi: 10.1016/j.fm.2006.02.005

Peres, C. M., Peres, C., Hernández-Mendoza, A., and Xavier Malcata, F. (2012). Review on fermented plant materials as carriers and sources of potentially probiotic lactic acid bacteria e with an emphasis on table olives. Trends Food Sci. Technol. 26, 31-42. doi: 10.1016/j.tifs.2012.01.006

Pino, A., Liotta, L., Randazzo, C. L., Todaro, A., Mazzaglia, A., De Nardo, F., et al. (2018). Polyphasic approach to study physico-chemical, microbiological and sensorial characteristics of artisanal Nicastrese goat's cheese. Food Microbiol. 70, 143-154. doi: 10.1016/j.fm.2017.09.005

Ranadheera, R. D. C. S., Baines, S. K., and Adams, M. C. (2010). Importance of food in probiotic efficacy. Food Res. Int. 43, 1-7. doi: 10.1016/j.foodres.2009.09.009

Randazzo, C. L., Fava, G., Tomaselli, F., Romeo, F. V., Pennino, G., Vitello, E., et al. (2011). Effect of kaolin and copper based products and of starter cultures on green table olive fermentation. Food Microbiol. 28, 910-919. doi: 10.1016/j.fm.2010.12.004

Randazzo, C. L., Rajendram, R., and Caggia, C. (2010). "Lactic acid bacteria in table olive fermentation," in Olives and Olive Oil in Health and Disease Prevention, eds R. Victor, Preedy, and Ronald Ross Watson (Oxford: Academic Press), 369-376. doi: 10.1016/B978-0-12-374420-3.00041-3

Randazzo, C. L., Ribbera, A., Pitino, I., Romeo, F. V., and Caggia, C. (2012). Diversity of bacterial population of table olives assessed by PCR-DGGE analysis. Food Microbiol. 32, 87-96. doi: 10.1016/j.fm.2012.04.013

Randazzo, C. L., Todaro, A., Pino, A., Corona, O., and Caggia, C. (2017). Microbiota and metabolome during controlled and spontaneous fermentation of Nocellara Etnea table olives. Food Microbiol. 65, 136-148. doi: 10.1016/j.fm.2017.01.022

Randazzo, C. L., Todaro, A., Pino, A., Pitino, I., Corona, O., Mazzaglia, A., et al. (2014). Giarraffa and Grossa di Spagna naturally fermented table olives: effect of starter and probiotic cultures on chemical, microbiological and sensory traits. Food Res. Int. 62, 1154-1164. doi: 10.1016/j.foodres.2014.05.056

Ruiz-Barba, J. L., Cathcart, D. P., Warner, P. J., Jiménez-Diáz, R. (1994). Use of Lactobacillus plantarum LPC010, a bacteriocin producer, as a starter culture in Spanish style green olive fermentation. Appl. Environ. Microbiol. 60, 2059-2064.

Sabatini, N., Mucciarella, M. R., and Marsilio, V. (2008). Volatile compounds in uninoculated and inoculated table olives with Lactobacillus plantarum (Olea europaea L., cv. Moresca Kalamata). LWT Food Sci. Technol. 41, 2017-2022. doi: 10.1016/j.lwt.2007.12.002

SAS (2001). User's Guide: Statistics, ver. 8.1. 2001. Cary, NC: SAS INST. INC.

Simpson, M. V., Smith, J. P., Dodds, K., Ramaswamy, H. S., Blanchfield, B., and Simpson, B. K. (1995). Challenge studies with Clostridium botulinum in a sous-vide spaghetti and meat sauce product. J. Food Prot. 58, 229-234. doi: 10.4315/0362-028X-58.3.229

Singleton, V. L. (1974). "Analytical fractionation of the phenolic substances of grapes and wines and some practical uses of such analyses," in Chemistry of Winemaking. Advances in Chemistry Series, Vol. 137, ed A. D. Webb (Washington, DC: American Chemical Society), 184-211.

Taormina, P. J. (2010). Implications of salt and sodium reduction on microbial food safety. Crit. Rev. Food Sci. Nutr. 50, 209-227. doi: 10.1080/10408391003626207

Tofalo, R., Perpetuini, G., Schirone, M., Fasoli, G., Suzzi, G., and Corsetti, A. (2014). Lactobacillus pentosus dominates spontaneous fermentation of Italian table olives. LWT Food Sci. Technol. 57, 710-717. doi: 10.1016/j.lwt.2014.01.035

Torriani, S., Felis, G. E., and Dellaglio, F. (2001). Differentiation of Lactobacillus plantarum, L. pentosus, and $L$. paraplantarum by recA gene sequence analysis and multiplex PCR assay with rec gene-derived primers. Appl. Environ. Microbiol. 67, 3450-3454. doi: 10.1128/AEM.67.8.34503454.2001

Valerio, F., De Bellis, P., Lonigro, S. L., Morelli, L., Visconti, A., and Lavermicocca, P. (2006). In vitro and in vivo survival and transit tolerance of potentially probiotic strains carried by artichokes in the gastrointestinal tract. Appl. Environ. Microbiol. 72, 3042-3045. doi: 10.1128/AEM.72.4.3042-3045.2006

Ventura, M., Canchaya, C., Meylan, V., Klaenhammer, T. R., and Zink, R. (2003). Analysis, characterization, and loci of the tuf genes in Lactobacillus and Bifidobacterium species and their direct application for species identification. Appl. Environ. Microbiol. 69, 6908-6922. doi: 10.1128/AEM.69.11.6908-6922.2003

World Health Organisation (WHO) (2003). Diet, Nutrition and the Prevention of Chronic Disease Report of a Joint WHO/FAO Expert Consultation. Technical Report Series No, Geneva. 916.

World Health Organisation (WHO) (2007). Reducing Salt Intake in Populations. Report of a WHO Forum and Technical Meeting. WHO Document Publication Services, Geneva.

Zinno, P., Guantario, B., Perozzi, G., Pastore, G., and Devirgiliis, C. (2017). Impact of $\mathrm{NaCl}$ reduction on lactic acid bacteria during fermentation of Nocellara del Belice table olives. Food Microbiol. 63, 239-247. doi: 10.1016/j.fm.2016.12.001

Conflict of Interest Statement: The authors declare that the research was conducted in the absence of any commercial or financial relationships that could be construed as a potential conflict of interest.

Copyright (c) 2018 Pino, De Angelis, Todaro, Van Hoorde, Randazzo and Caggia. This is an open-access article distributed under the terms of the Creative Commons Attribution License (CC BY). The use, distribution or reproduction in other forums is permitted, provided the original author(s) and the copyright owner are credited and that the original publication in this journal is cited, in accordance with accepted academic practice. No use, distribution or reproduction is permitted which does not comply with these terms. 